\title{
BARGAINING AND REPUTATION IN SEARCH MARKETS
}

\author{
ALP E. ATAKAN AND MEHMET EKMEKCI
}

\begin{abstract}
.
In a two-sided search market agents are paired to bargain over a unit surplus. The matching market serves as an endogenous outside option for agents in a bargaining relationship. Behavioral agents are (strategically inflexible) commitment types that demand a constant portion of the unit surplus. The steady state frequency of behavioral types in the market is determined in equilibrium. We show, even if behavioral types are negligible, they substantially effect the terms of trade and efficiency. In an unbalanced market where the entering flow of one side is short, bargaining follows equilibrium play in a bargaining game with one-sided reputation, the terms of trade are determined by the commitment types on the short side, and commitment types improve efficiency. In a balanced market where the entering flows of the two sides are equal, bargaining follows equilibrium play in a bargaining game with two-sided reputation and commitment types cause inefficiency. An inefficient equilibrium with persistent delays and break-ups is constructed. The magnitude of inefficiency is determined by the inflexible demands of the commitment types and is independent of the fraction of the commitment types entering the market.
\end{abstract}

Keywords: .

JEL Classification Numbers: .

\section{Introduction And Related Literature}

Classical price theory suggests that the impact of a small number of "behavioral" agents on aggregate equilibrium variables in a large market should also be small. Recent research shows that outcomes of bilateral dynamic interactions, where agents are rational (i.e., not behavioral), can be drastically different than outcomes of bilateral interactions where there is even a small amount of incomplete information concerning the rationality of the agents 1 These two insights suggest a tension between the

Date: First draft, March, 2008. This revision, February, 2009.

${ }^{1}$ See, Milgrom and Roberts (1982), Kreps and Wilson (1982), Fudenberg and Levine (1989) and Fudenberg and Levine (1992) for demonstrations of this phenomenon in repeated games; or Myerson 
impact of behavioral agents in bilateral relationships and aggregate market forces if a large market is an agglomeration of many bilateral bargaining relationships 2 In this paper we analyze how the impact of behavioral agents in bilateral bargaining interacts with aggregate forces to determine the equilibrium outcome of a large search market. In particular, we explore whether aggregate market forces overwhelm the impact of a small number of behavioral agents or, alternatively, whether the presence of even a small number of behavioral agents translates into a large effect on the equilibrium outcome.

Bilateral bargaining outcomes are highly sensitive to the outside options as well as incomplete information about the "types" of the bargaining agents. Consider a two player alternating offers bargaining game over a unit surplus where the time between offers is arbitrarily small. Without incomplete information, the unique perfect equilibrium is the Rubinstein (1982) outcome (see also Shaked and Sutton (1984), Sutton (1986) and Perry and Reny (1993)).

Suppose instead that there is incomplete information about the type of player 1. In particular, if agent 1 is potentially a (strategically inflexible) "commitment" type that insists on portion $\theta_{1}$ of the bargaining surplus, and player 2 is a normal type with certainty, then player 1 obtains $\theta_{1}$ and player 2 receives $1-\theta_{1}$ in any perfect equilibrium, even if the probability that player 1 is a commitment type is arbitrarily small (the one-sided reputation result of Myerson (1991)).

In addition to player 1 , suppose that there is also incomplete information about the type of player 2 . In particular, if both players are potentially commitment types that demand $\theta_{1}$ and $\theta_{2}$, then a war of attrition ensues, and the unique equilibrium payoff profile is inefficient with the "weak" agent (agent $i$ ) receiving $1-\theta_{j}$ and the "strong" agent receiving strictly less than $\theta_{j}$ (the two-sided reputation result of Abreu and Gul (2000)). However, now suppose that both players have access to an outside option. If agent $i$ 's outside option exceeds $1-\theta_{j}$ and $j$ 's outside option is less that $1-\theta_{i}$, then player $i$ never yields to $j$, eliminating the incentive for $j$ to build a reputation and the outcome is identical to the one-sided incomplete information case where $i$ receives $\theta_{i}$

(1991), Kambe (1999), Abreu and Gul (2000), Compte and Jehiel (2002), or Abreu and Pearce (2007) for examples in bilateral bargaining.

${ }^{2}$ The labor market and the housing market are particular examples of such markets. For economic models of such markets see, Rubinstein and Wolinsky (1990, 1985), Serrano and Yosha (1993), or Osborne and Rubinstein (1990) for a more complete overview. 
and $j$ receives $1-\theta_{i}$ (Lemma 1). Moreover, if both agents' outside options dominate yielding to the commitment type, then the incentive to build a reputation is entirely eliminated and the unique equilibrium is again the Rubinstein outcome (Compte and Jehiel (2002)).

As outlined above, the outcome of bilateral bargaining depends heavily on the distribution over agent types while the distribution over agent types is an endogenous variable determined in a market equilibrium. In turn, the market equilibrium may depend on bargaining outcomes: agent types that are traded infrequently, but that nevertheless obtain high values, are plentiful; while agent types that are traded very frequently, or that have very low values, are scarce. Also, bargaining outcomes depend crucially on the outside options of agents, and again, outside options are endogenous variables determined in equilibrium.

To address the aforementioned issues of endogeneity, this paper presents a twosided search model where agents are paired to bargain over a unit surplus. The two sides of the market can be thought of as buyers and sellers of a homogeneous good. The matching market serves as the endogenous outside option for agents in a bargaining relationship. In each period a constant measure of agents enter the market. Agents exit the market through successfully making a trade or they leave voluntarily because there are no profitable trading opportunities in the market. A fraction of the entering population on each side is comprised of commitment types. The steady state frequency of behavioral types in the market is determined in equilibrium and if the entering fraction of behavioral types is small, then so is the equilibrium frequency of behavioral types.

A central finding of this paper is that even a negligible number of behavioral agents significantly affect equilibrium outcomes 3 Compte and Jehiel (2002) demonstrated that if the outside options of the normal types are sufficiently high, then commitment types have no effect on bargaining outcomes. In the market analyzed here, however, the endogenous outside options of the normal agents are never large enough to deter the commitment types. In equilibrium, some normal types always trade with commitment types. This, in turn, makes normal agents in the market excessively greedy in

\footnotetext{
${ }^{3}$ In all the results described below the time between offers in the bargaining stage is taken as arbitrarily small.
} 
bargaining. Consequently, even if behavioral types are negligible, they substantially effect the terms of trade and the efficiency of the aggregate market.

Although behavioral agents always have an impact on equilibrium outcomes, the nature of their effect depends on aggregate forces. The paper focuses on two cases: an unbalanced market where the entering flow of one side is short; and a balanced market where the entering flows of the two sides are equal. Unbalanced markets entail onesided reputation building; and balanced markets entail two-sided reputation building, in equilibrium. Note that commitment types are present on both sides regardless of whether the market is balanced or unbalanced. Nevertheless, in an unbalanced market, only the short-side chooses to imitate the commitment types, whereas, in a balanced market, both sides imitate the commitment types. In particular:

(i) In an unbalanced market a fraction of the agents in the long side of the market must be leaving the market without trading in any steady state. Consequently, aggregate flows ensure that the outside option of the long-side is compatible with the demands of the commitment types while the outside option of the short-side is incompatible. However, if the short-side's outside option is incompatible and the long-side's outside option is compatible with the commitment type demands, then equilibrium play in the bargaining stage involves one-sided reputation building by agents on the short-side.

(ii) In balanced markets the effects of the commitment types are most pronounced. In equilibrium, aggregate forces ensure that the outside options of both sides are compatible with the inflexible demands of the commitment types. So the magnitude of inefficiency is determined by the inflexible demands of the commitment types. We construct an equilibrium where: The normal types play a war of attrition and always trade. A normal type of side $i$ always trades with a commitment types of side $j$. A normal type of side $j$ optsout against the commitment type with positive probability. Bargaining is inefficient and the inefficiency is caused by delay and break-ups. In sharp contrast to existing literature (Abreu and Gul (2000) and Compte and Jehiel (2002)), as the fraction of commitment types entering the market becomes small, the inefficiency (manifested as delay) persists.

The paper is organized as follows: section 2 describes the model; section 3 solves the baseline economy without any commitment types; section 4 analyzes the bargaining 
stage-game and presents the required interim results, section 5 presents the main results and section 6 concludes. All proofs that are not in the main text are in the appendix.

\section{THE MODEL}

In each period agents belonging to two classes $i \in\{1,2\}$ (for example, buyers and sellers of a homogeneous good) enter a matching market. Mass $L_{i}$ of agents enter from each class. Of the class $i$ agents entering the market a fraction $z_{i}$ are commitment types and the remainder $1-z_{i}$ are normal types. We refer to a normal type agent of class 1 as player 1 or him and to a normal type agent of class 2 as player 2 or her.

In each period a portion of the unmatched agents in the market are randomly paired with a potential trading partner from the opposite class to play a bilateral bargaining game. Unmatched agents that are not paired can wait for $t_{\text {search }} / \Delta$ periods for another chance to be paired with a bargaining partner, or can choose to leave the market and receive an exogenous outside option. In each bilateral bargaining game a unit surplus is available for division between the paired agents. Agents only receive utility if they can agree on the division of the unit surplus. If two matched agents agree on the division of the unit surplus, then they trade. Agents that trade permanently leave the market. The division of the unit surplus is determined in an alternating offers bargaining game with the possibility of opting-out.

2.1. The Bargaining Stage-Game. When two agents are matched they play an alternating offers bargaining game over a unit surplus denoted $\Gamma$. In odd periods the agent from class 1 is the proposer and in even periods the agent from class 2 is the proposer. Bargaining between the two agents continues until there is agreement or one of the two agents opts-out from the bargaining game. The proposer can make an offer, opt-out and join the unmatched population, or opt-out and leave the market to take the exogenous outside option. If the proposer chooses to make an offer, then he/she proposes a division of the unit surplus. After an offer, the responder can accept the offer, reject the offer, opt-out and join the unmatched population, or opt-out and leave the market. If after an offer by agent $j$, if agent $i$ rejects the offer, then the agents wait for a period of length $\Delta$ after which time agent $i$ is the new proposer. If any agent opts-out, then either each agent chooses to join the pool of unmatched agents after $t_{\text {search }} / \Delta \geq 1$ periods, i.e, after $t_{\text {search }}$ units of time. Or, the agent can 
leave the market and receive the exogenous outside option. The extensive form the the bargaining stage-game is given in Figure 1 .

The period length $\Delta$ measures the amount of time it takes to formulate a counteroffer. So, it is a measure of with-in bargaining frictions. If $\Delta \approx 0$, then agents are able to make offers almost instantaneously. The parameter $t_{\text {search }}$ measures the amount of time it takes to generate a new bargaining opportunity and is a proxy for the magnitude of search frictions. If $t_{\text {search }} \approx 0$, then agents are able to generate new bargaining partners almost instantaneously.

2.2. Agents. The market is comprised of two classes of agents. Also, each class is comprised of normal type agents and commitment type (or stubborn) agents.

2.2.1. Normal types. Normal type agents belonging to class $i$ are impatient with instantaneous rates of time preference $r_{i}$. Consequently, if the normal type agent of class $i$ reaches an agreement that gives this player $y_{i}$ units of surplus at period $s$, then his utility $u_{i}=y_{i} e^{-r_{i} \Delta s}$. Also, $\delta_{i}=e^{-r_{i} t_{\text {search }}}$ denotes the time cost of opting-out and searching for a new partner. The utility from taking the exogenously given outside for all agents is equal to $\delta_{i} x$.

2.2.2. Commitment types. A commitment type is assumed to insist on share $\theta_{i}$ of the unit surplus and reject any offer that gives him less than $\theta_{i}$. The commitment types are incompatible, in particular, $\theta_{i}+\theta_{j}>1$. Also, the commitment types never opt-out as long as there is a positive probability that their opponent in their current match is the normal type, and opt-out or leave the market, otherwise. Consequently, the probability that two commitment types remain in a bargaining relationship forever is zero. The commitment types decide whether to leave the market or not using the same payoff calculation as the normal types 4

2.3. The Pool of Unmatched Agents and Matching. Let $N_{i}^{s}$ denote the measure of unmatched normal types of class $i$ in the matching market in period $s$ and let $C_{i}^{s}$ denote the measure of unmatched commitment types of class $i$ in the matching market in period $s$. Also, let $n_{i}^{s}=\frac{N_{i}^{s}}{N_{i}^{s}+C_{i}^{s}}$ and $c_{i}^{s}=\frac{C_{i}^{s}}{N_{i}^{s}+C_{i}^{s}}$, that is $n_{1}^{s}$ is the proportion

\footnotetext{
${ }^{4}$ This is a much stronger assumption than we need on the preferences of the commitment types. All the result go through under the following behavioral assumption: if the probability of being traded is strictly positive and if the expected time at which a trade occurs is finite, then there exists an $x^{*}$ such that the commitment types strictly prefer to remain in the market for all $x<x^{*}$.
} 


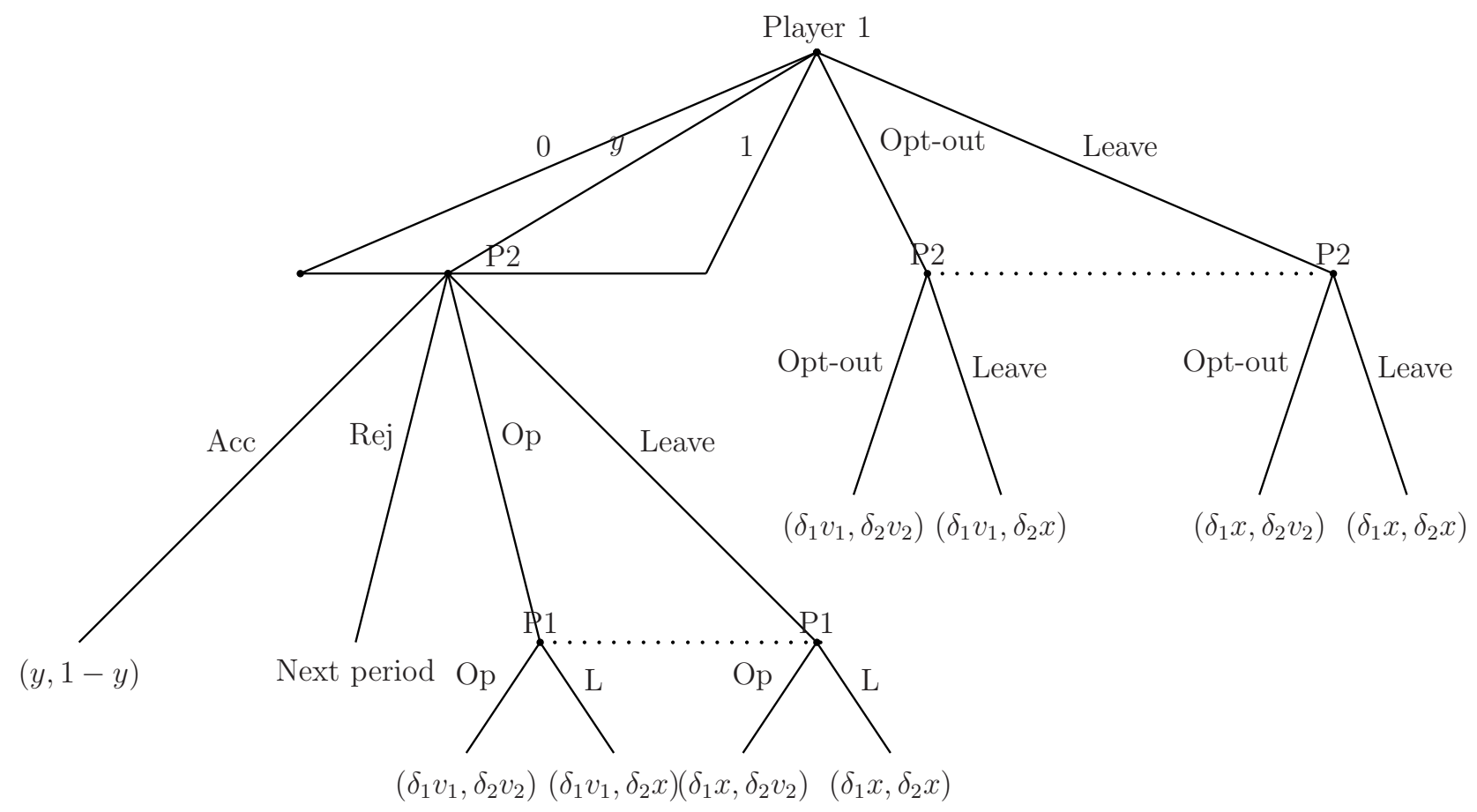

FiguRE 1. This depicts the bargaining game in any odd period where player 1 speaks first. He can make any offer $y \in[0,1$,$] , opt-out and$ return to the unmatched population or leave the market. $v_{i}$ denotes the value to player $i$ of being unmatched in the market. Player 1 receives $\delta_{1} v_{1}$ if he returns to the unmatched population and receives $\delta_{1} x$ if he leaves the market. Player 2 speaks second and can accept player 1's offer, reject the offer, opt-out or leave the market. If she rejects then the game progresses to the next period where the roles are reversed.

of normal types among unmatched class 1 agents in period $s$ and similarly $c_{2}^{s}$ is the proportion of commitment types among unmatched class 2 agents in period $s$. Also, let $m_{i}^{s}=\min \left\{1, \frac{N_{j}^{s}+C_{j}^{s}}{N_{i}^{s}+C_{i}^{s}}\right\}$. If in the pool of unmatched agents the measure of agents from the two classes is equal, then the market tightness (which is the inverse of the "queue length") parameter $m_{1}^{s}=m_{2}^{s}=1$. Otherwise, since one side of the market is larger, these agents are rationed and the market tightness for this side is less than one. The pool of unmatched agents in period $s$ is comprised of agents that entered the market in period $s$; agents whose bargaining arrangement dissolved as a result of one of the parties' opting out in period $s-t_{\text {search }} / \Delta$; and agents who, in period 
$s-t_{\text {search }} / \Delta$ were not paired with a bargaining partner and who chose to remain in the market. An agent of class $i$ is matched with a normal type of class $j$ with probability $n_{j}^{s}$ and the commitment type with probability $c_{j}^{s}$, in period $s$. Consequently, measure $m_{1}^{s} N_{1}^{s} n_{2}^{s}=m_{2}^{s} n_{1}^{s} N_{2}^{s}$ of (normal, normal) pairs; measure $m_{1}^{s} C_{1}^{s} n_{2}^{s}=m_{2}^{s} c_{1}^{s} N_{2}^{s}$ of (commitment, normal) pairs; measure $m_{1}^{s} N_{1}^{s} c_{2}^{s}=m_{2}^{s} n_{1}^{s} C_{2}^{s}$ of (normal, commitment) pairs; and measure $m_{1}^{s} C_{1}^{s} n_{2}^{s}=m_{2}^{s} c_{1}^{s} C_{2}^{s}$ (commitment, commitment) pairs are created, in each period $s$.

2.4. Strategies. Let $h_{t}$ denote a history for agent $i$ and let $H_{t}$ denote the set of all histories for player $i$ at time $t$. In the definition of history, time $t$ refers to the $t$ periods from the time that player $i$ entered the market, i.e, period 1 is the first active period for player $i$. A strategy for player $i, \sigma_{i}: H_{t} \rightarrow[0,1] \cup\{$ opt - out, leave $\}$ if at period $t$ player $i$ is making an offer and $\sigma_{i}: H_{t} \rightarrow\{$ accept, reject, opt-out, leave $\}$ if at period $t$ player $i$ is responding. Also, at the end of each period, agents in the pool of unmatched agents can leave the market and take their exogenous outside option, or choose to stay in the market until the next period. Consequently, at a history where player i needs to choose whether to leave or stay, $\sigma_{i}: H_{t} \rightarrow\{$ leave, stay $\}$. A behavior strategy is similarly defined but has the player randomizing over the action choices. In the paper we focus on symmetric strategies, i.e., we assume that agents of the same type and class use the same strategy. Also, we assume that players condition their choices in the bargaining stage game only on the history in their current match.

A belief for agent $i$ is a function $\mu_{i}: H_{t} \rightarrow[0,1]$ that gives the probability that agent $i$ places on his rival being the commitment type. Assume that in the first period of the match, before any action has been taken, agent $i$ 's prior belief coincides with the probability of drawing a commitment type from the general population. That is, the prior belief is the same as the frequency of commitment types in the unmatched population.

2.5. Steady State. The analysis focuses on the steady state of the system. In each period the measure of agents leaving the market by successfully consummating a trade or trough voluntary exit equals the inflow of traders into the market (pool of unmatched agents) resulting either from a break-up or through new entry. In each period unmatched buyers and sellers are matched with each other randomly. The probability that a buyer is matched with a rational seller is equal to the frequency of 
rational sellers in the steady state. Consequently, the steady state equations for the market are as follows

$$
\begin{aligned}
\left(1-z_{1}\right) L_{1} & =N_{1} m_{1}\left(n_{2} p_{n n}+c_{2} p_{n c}\right)+E_{1}^{n} \\
z_{1} L_{1} & =C_{1} m_{1}\left(n_{2} p_{c n}+c_{2} p_{c c}\right)+E_{1}^{c} \\
\left(1-z_{2}\right) L_{2} & =n_{2} m_{2}\left(N_{1} p_{n n}+C_{1} p_{c n}\right)+E_{2}^{n} \\
z_{2} L_{2} & =c_{2} m_{2}\left(N_{1} p_{n c}+C_{1} p_{c c}\right)+E_{2}^{c}
\end{aligned}
$$

where $p_{n n}$ is the total probability that two normal types who are matched would eventually trade with each other and $E_{1}^{n}$ is the measure of class 1 normal types leaving the market without trading at the end of the period. The vector of match probabilities $p$, as well as, the vector of outflows $E$ are obtained from the (equilibrium) strategy profiles.

2.6. Equilibrium. Let $\Gamma(\Delta, x, c, v)$ denote the bargaining stage-game where the time between offers is $\Delta$, the exogenous outside option is worth $\delta_{i} x$ to player $i$, opting out to the unmatched population is worth $\delta_{i} v_{i}$ to player $i$, and the initial belief that player $i$ 's opponent is the commitment type, $\mu_{i}\left(h_{0}\right)$ is equal to $c_{i}$. Let $U_{i}(\sigma)$ denote the payoff that player i, i.e., a normal type of class i, obtains in the bargaining stage game conditional on facing player $j$. Let $v_{i}(\sigma)$ denote the value for player $i$ of being in the unmatched population.

A search equilibrium $\sigma$ is comprised of a strategy $\sigma_{k}$ for each agent type; a belief function $\mu_{k}$ for each type of agent; and steady state measures $\left(N_{1}, C_{1}, N_{2}, C_{2}\right)$, that are mutually compatible. More precisely, the strategy profile $\left(\sigma_{1}, \sigma_{2}\right)$ and the belief profile $\left(\mu_{1}, \mu_{2}\right)$ comprises a perfect equilibrium in the bargaining stage-game $\Gamma(\Delta, x, c, v)$, where $c_{i}$ is the equilibrium frequency of class $i$ commitment types and $v_{i}$ is the equilibrium value for player $i$. Also, the market remains in steady state, i.e., Equations (11) through (44) are satisfied given that the match probabilities are derived from the equilibrium strategy profile $\left(\sigma_{1}, \sigma_{2}\right)$.

\section{Baseline Economy with no Commitment Types}

First, before introducing commitment types, we study the baseline economy with only rational agents $\left(z_{1}=z_{2}=0\right)$. In this economy, equilibrium play in the bargaining 
stage-game unfolds according to the complete information alternating offers bargaining model of Rubinstein (1982). Recall that $\Delta \leq t_{\text {search }}$, that is, once in a bargaining relationship it takes less time to make a counter offer than to opt-out and search for a new bargaining partner. This implies, with only rational agents, players never opt-out, after any history. So, play is identical to an alternating offers bargaining game without opt-outs which has the Rubinstein outcome as its unique equilibrium. Define $u_{1}^{*}(\Delta) \equiv \frac{1-e^{-r_{2} \Delta}}{1-e^{-\left(r_{1}+r_{2}\right) \Delta}}$ and $u_{2}^{*}(\Delta) \equiv \frac{e^{-r_{2} \Delta}\left(1-e^{-r_{1} \Delta}\right)}{1-e^{-\left(r_{1}+r_{2}\right) \Delta}}$ as the Rubinstein payoffs.

In each period, an equal number of agents from class $i$ and $j$ leave the market as a result of successful trades. This is because all trade occurs in pairs. If the market is unbalanced $\left(L_{i}>L_{j}\right)$, then there are more class $i$ agents entering the market than class $j$ agents in each period and, for the market to remain in steady state, some class $i$ agents must leave the market voluntarily without trading. So, in order to incentivize agents on the long side $i$, equilibrium values for class $i$ must equal the exogenous outside option $x$, i.e., $v_{i}=x$. However, since each agent $i$ receives a substantial portion of the unit pie in the bargaining stage game, the market tightness $m_{i}$ for side $i$ must be sufficiently smaller than 1, (or in alternative terminology, the queue length, $\frac{1}{m_{i}}$, must be sufficiently long) in order for agent $i$ 's value to equal $x$. Alternatively, if the markets are balanced $\left(L_{i}=L_{j}\right)$, then there is an equilibrium, with no queues on either side, in which each side receives their Rubinstein payoff. The following summarizes these results.

The Complete Information Benchmark. Suppose that $z_{1}=z_{2}=0$ and $x<$ $\min _{i} \delta_{i} u_{i}^{*}$. Then agents receive their Rubinstein payoffs in the bargaining stage game, $U_{i}(\sigma)=u_{i}^{*}$, in any search equilibrium $\sigma$. Also,

(i) If $L_{i}>L_{j}$, then $v_{i}(\sigma)=x$ and $v_{j}(\sigma)=u_{j}^{*}$ in any search equilibrium $\sigma$,

(ii) If $L_{1}=L_{2}$, then $v_{i}(\sigma)=u_{i}^{*}$ and $x \leq v_{j}(\sigma) \leq u_{i}^{*}$ in any search equilibrium $\sigma$. Also, there is a search equilibrium $\sigma$ such that $v_{i}(\sigma)=u_{i}^{*}$ and $v_{j}(\sigma)=u_{i}^{*}$.

Proof. Follows from Rubinstein (1982), Shaked and Sutton (1984) or Osborne and Rubinstein (1990). For an argument see Appendix A.

\section{The Bargaining Stage-game}

This section presents results for the bargaining stage game used in the analysis of the full economy. Lemma 1, Lemma 2 and Lemma 3 take as given the vector of outside 
options, $v=\left(\delta_{1} v_{1}, \delta_{2} v_{2}\right)$, and the vector of commitment type probabilities $c=\left(c_{1}, c_{2}\right)$ and characterize perfect Bayesian equilibria for the bargaining stage-game $\Gamma(\Delta, c, v)$. Lemma 1 considers a situation where player 1's outside option is incompatible with class 2 commitment types $\left(\delta_{1} v_{1}>1-\theta_{2}\right)$, while player 2's outside option is compatible with class 1 commitment types. In this situation, player 1 would rather opt-out than agree to trade with a commitment type. This eliminates player 2's incentive to mimic the commitment type. In particular, the lemma shows that player 2 will immediately reveal herself as rational. Once player 2 reveals herself, the continuation bargaining game is a game with one-sided incomplete information. In this continuation game player 1 can secure a payoff close to $\theta$, if $\Delta$ is sufficiently small, as first shown by Myerson (1991) 5 For the remainder of the paper we say player $i$ reveals rationality if $i$ accepts any offer other than $\theta_{i}$ in a period she responds, or proposes something other than $\theta_{i}$ in a period that she proposes.

Lemma 1 (One-sided reputation). Suppose that $c_{1}>0, c_{2}>0$. If $\delta_{1} v_{1}>1-\theta_{2}$ and $\delta_{2} v_{2}<1-\theta_{1}$, then

(i) Player 1 always proposes $\theta_{1}$,

(ii) Player 2 reveals rationality in period 1 or period 2 ,

(iii) There exists a constant $\kappa>0$, that is independent of $\Delta$ such that,

$$
\begin{array}{r}
\theta_{1}-\kappa\left(1-e^{-r \Delta}\right) \leq U_{1}(\sigma) \leq \theta_{1} \\
1-\theta_{1} \leq U_{2}(\sigma) \leq 1-\theta_{1}+\kappa\left(1-e^{-r \Delta}\right)
\end{array}
$$

where $r=\max \left\{r_{1}, r_{2}\right\}$,

in any perfect Bayesian equilibrium $\sigma$ of $\Gamma(\Delta, c, v)$.

Proof. The proof is given in Appendix B. Here is a sketch of the argument. If player 2 is known to be rational and player 1 is not, then player 1 receives a payoff close to $\theta_{1}$ (player 1's one-sided reputation payoff). If player 1 is known to be rational and player 2 is not, then the players get their Rubinstein payoffs. This is because player 1's outside option precludes player 2 from building a reputation. Player 2 reveals rationality at the latest by some period $T$. So, player 1 will opt-out with certainty in period $T+1$. Consequently, if player 1 ever makes an offer different than $\theta_{1}$, then there

\footnotetext{
${ }^{5}$ An analysis of the bargaining game with one-sided incomplete information is provided in Appendix C
} 
is a well defined last period in which player 1 makes this offer. If Player 1 always offers $\theta_{1}$, then player 2 can do no better than to reveal rationality immediately. Observe player 1 never accepts $\theta_{2}$. So a trade occurs only if player 2 accepts $\theta_{1}$ or if player 2 reveals rationality, i.e., plays an action other than $\theta_{2}$. Consequently, not revealing rationality only delays player 2 receiving the payoff from revealing rationality. In the last period player 1 is supposed to offer something different than $\theta_{1}$, denoted period $S$, player 1 can instead offer $\theta_{1}$, get player 2 to reveal rationality at the latest in the following period and obtain a payoff close to $\theta_{1}$. Consequently, player 1 will not offer anything but $\theta_{1}$ in period $S$. However, this implies player 1 will always offer $\theta_{1}$, player 2 will reveal rationality at the latest by period 2 , and player 1 will obtain his one-sided reputation pay-off that is close to $\theta_{1}$.

Lemma 2 turns attention to the case where both agents outside options are worse than trading with the commitment type. In this case both players would trade, even if they believe their opponent to be the commitment type, rather than take the outside option. This game is identical to the bargaining game analyzed by Abreu and Gul (2000). Let,

$$
\begin{aligned}
\lambda_{i} & \equiv \lim _{\Delta \rightarrow 0}\left(\lambda_{i}(\Delta)=\frac{\left(1-e^{-r_{j} \Delta}\right)\left(1-\theta_{i}\right)}{\Delta\left(\theta_{i}+\theta_{j}-1\right)}\right)=\frac{r_{j}\left(1-\theta_{i}\right)}{\left(\theta_{i}+\theta_{j}-1\right)} \\
T_{i} & \equiv-\ln c_{i} / \lambda_{i} \\
T & =T_{i} \min \left\{\frac{T_{j}}{T_{i}}, 1\right\} \text { and } \\
b_{i} & \equiv c_{i} c_{j}^{-\lambda_{i} / \lambda_{j}} \text { for } T_{i}>T_{j} .
\end{aligned}
$$

Abreu and Gul (2000) showed that all perfect equilibria of the bargaining game converge to a war of attrition where each agent reveals their rationality with constant hazard rate $\lambda_{i}$, both agents complete their revealing at time $T$, and the "weaker" agent, i.e, the agent $i$ with the larger $T_{i}$, will concede with positive probability $b_{i}$ at time zero. In the war of attrition, after time zero, both agents are indifferent between revealing rationality immediately to their opponent or continuing to resist. This implies that the payoff to the normal type is equal to the payoff obtained by yielding immediately to the commitment type $1-\theta_{j}$ after time zero. Consequently, for small $\Delta$, the bargaining game payoff of the strong player is approximately $\left(1-b_{j}\right) \theta_{i}+b_{j}\left(1-\theta_{j}\right)$. These findings are summarized in the following lemma. 
Lemma 2 (Two-sided reputation). Suppose that $c_{1}>0, c_{2}>0$. If $\delta_{1} v_{1}<1-\theta_{2}$ and $\delta_{2} v_{2}<1-\theta_{1}$, then there exists $\kappa>0$, independent of $\Delta$, such that

$$
\left|U_{i}(\sigma)-\left(\left(1-b_{j}\right) \theta_{i}+b_{j}\left(1-\theta_{j}\right)\right)\right| \leq \kappa\left(1-e^{-r \Delta}\right)^{1 / 2}
$$

in any perfect Bayesian equilibrium $\sigma$ of $\Gamma(\Delta, c, v)$

Proof. See Compte and Jehiel (2002) Proposition 3 or Abreu and Gul (2000) Proposition 4.

Lemma 3 considers a situation where both normal agents' outside options exceed the payoff from trading with their opponents commitment types. Under this scenario the incentive to mimic the commitment type if eliminated for both players since their opponent never yields to the commitment type. However, once both players reveal rationality, the unique perfect equilibrium of the bargaining game leads to the Rubinstein outcome. This result, established in Compte and Jehiel (2002), is summarized in the following lemma.

Lemma 3. Suppose that $c_{1}>0, c_{2}>0$. If $\delta_{1} v_{1}>1-\theta_{2}$ and $\delta_{2} v_{2}>1-\theta_{1}$, then $U_{1}(\sigma)=u_{1}^{*}(\Delta)$ and $U_{2}(\sigma)=u_{2}^{*}(\Delta)$ in any perfect Bayesian equilibrium $\sigma$ of $\Gamma(\Delta, c, v)$

Proof. See Compte and Jehiel (2002) Proposition 5.

\section{Main Results}

This section presents the main reputation results for the model with commitment types. All the results focus on the case where the exogenous outside option is small ( $x$ is close to zero) so that the sole purpose of the exogenous outside option is to stop agents that have low payoff in equilibrium from clogging the market. Also, the time between offers in the bargaining stage is assumed as arbitrarily small. Three main results are presented. Theorem 1 considers an unbalanced market and shows that equilibrium play is characterized by one-sided reputation building. Theorem 2 considers a balanced market and shows that the equilibrium outside options of all normal types are compatible with the inflexible demands of commitment types. Consequently, inefficiency is substantial. Corollary 1 and Corollary 2 generalize these results to multiple commitment types on both sides of the market. The third main result, Theorem 3, again considers a balanced market and constructs an equilibrium. 
In this equilibrium, the two rational agents play a war of attrition, there are delays in reaching an agreement between the rational agents, and opt-outs occur on the equilibrium path. Corollary 3 presents the comparative statics for Theorem 3 and shows, even at the limit with complete rationality, inefficiencies and delay remain substantial in equilibrium.

5.1. Unbalanced markets and One-Sided Reputation. The following theorem considers a situation where there are more normal class 2 agents entering the market looking for a trade than there are agents available on side 1 . In a steady state a portion of the class 2 agents must leave the market without trading by taking their exogenous outside option. In order to incentivize player 2 to choose her exogenous outside option, her value from remaining in the market, $\delta_{2} v_{2}$, must be at most equal to $x$. This implies, however, that player 2 must be willing to trade with any class 1 commitment type $\left(1-\theta_{1}>x\right)$. The following result also demonstrates that agent 1 's equilibrium value dominates conceding to his opponent's commitment type, $\delta_{1} v_{1}>1-\theta_{2}$, in any equilibrium. Hence the bargaining stage-game that the agents play is identical to the case covered by Lemma 1. Consequently, player 1 always mimics the commitment type, player 2 reveals rationality immediately, and agent 1's equilibrium value is close to $\theta_{1}$.

Theorem 1. Suppose that $L_{2}\left(1-z_{2}\right)>L_{1}$ and $\delta_{1} \theta_{1}>1-\theta_{2}$. If $0 \leq \Delta<\bar{\Delta}$ and $0<x<\bar{x}$, then $v_{2}(\sigma)=x$ and $\delta_{1} v_{1}(\sigma)>1-\theta_{2}$, and,

(i) In the bargaining stage-game player 1 always proposes $\theta_{1}$,

(ii) Player 2 reveals rationality in period 1 or period 2,

(iii) There exists a constant $\kappa>0$, that is independent of $\Delta$ such that,

$$
\begin{aligned}
& \theta_{1}-\kappa\left(1-e^{-r \Delta}\right) \leq U_{1}(\sigma) \leq \theta_{1} \\
& 1-\theta_{1} \leq U_{2}(\sigma) \leq 1-\theta_{1}+\kappa\left(1-e^{-r \Delta}\right) \\
& \theta_{1}-\kappa\left(1-e^{-r \Delta}\right) \leq v_{1}(\sigma) \\
& \text { where } r=\max \left\{r_{1}, r_{2}\right\} \text {, }
\end{aligned}
$$

in any search equilibrium $\sigma$.

The equilibrium in Theorem 1 contrasts with the Complete Information Benchmark (item $i$ ). This is because player 2 is always willing to trade with class 1 commitment 
types. This implies that there can never be an equilibrium where player 1 does not mimic a commitment type. More subtly the equilibrium behavior also contrasts with the two-side reputation result presented in Lemma 2. Player 1's equilibrium payoff strictly exceeds the inflexible demands of class 2 commitment types precluding player 2 from building a reputation. In particular the theorem shows,

(i) Player 1's equilibrium value, $\theta_{1}-\kappa\left(1-e^{-r \Delta}\right)$, strictly exceeds his equilibrium payoff without commitment types $u_{1}^{*}(\Delta)$, and also the commitment type demand $1-\theta_{2}$. This implies that only player 1 builds a reputation.

(ii) Since the market tilts the bargaining power in the bargaining stage game towards player 1 , the queue length required to make player 2 willing to take the exogenous outside option is reduced and consequently the overall efficiency of the market is improved.

(iii) The inefficiency in the bargaining stage is minimal. On the equilibrium path player 2 immediately reveals rationality and the number of periods of delay, in a game with one-sided incomplete information, is at most $\kappa$.

proof of Theorem 1. In the following development we assume that $x$ is sufficiently small as needed.

Step 1. In any equilibrium $v_{2}(\sigma)=x$ and consequently $m_{2}<1$ and $m_{1}=1$.

In order for the steady state equations to hold some of the class 2 agents must be leaving the market without trading. This implies that player 2's value $v_{2}(\sigma)=x$. In any bargaining stage game, player 2 can guarantee $1-\theta_{1}$ so in any equilibrium $v_{2} \geq m_{2}\left(1-\theta_{1}\right)+\left(1-m_{2}\right) \delta_{2} v_{2}$. Consequently, $x=v_{2} \geq \frac{m_{2}\left(1-\theta_{1}\right)}{1-\delta_{2}\left(1-m_{2}\right)}$. This implies that $m_{2} \leq \frac{x\left(1-\delta_{2}\right)}{\left(1-\theta_{1}\right)-\delta_{2} x}$. Consequently, $m_{2}$ is arbitrarily close to zero for $x$ small. However, $m_{2}<1 \Rightarrow m_{1}=1$.

Step 2. In any equilibrium $C_{1} \geq L_{1} z_{1}$ and $C_{2}=z_{2} L_{2}$.

$C_{1} \geq L_{1} z_{1}$ because $L_{1} z_{1}$ is the number of class 1 commitment agents that enter the market in each period. Any class 2 commitment type does strictly worse than player 2. This is because player 2 can do at least as well as the commitment type against player 1 by using the same strategy as the commitment type. Also, player 2 can trade with class 1 commitment types and obtain $1-\theta_{1}$ in these meetings. If the value of player 2 is less than or equal to $x$, then the payoff for a class 2 commitment type is strictly less than $x$. Consequently, all of these types, who are in the unmatched 
population at the end of a period, will choose to voluntarily exit instead of waiting $t_{\text {search }} / \Delta$ periods for a possible match. So $C_{2}=z_{2} L_{2}$.

Step 3. Take a sequence of $x^{k} \rightarrow 0$ and let $\sigma^{k}$ denote a search equilibrium when the exogenous outside option is equal to $x^{k}$. For any sequence of search equilibria $\sigma^{k}$, $N_{2}^{k} \rightarrow \infty, n_{2}^{k} \rightarrow 1$ and $c_{2}^{k} \rightarrow 0$. Also, there exists $\epsilon>0$ such that, for all $x^{k}<\bar{x}$, $c_{1}^{k} \geq \epsilon$.

If $x \rightarrow 0$, then $m_{2} \leq \frac{x\left(1-\delta_{2}\right)}{\delta_{2}\left(1-\theta_{1}\right)-x} \rightarrow 0$. Also $C_{1}+N_{1} \geq L_{1}$ and $C_{2}=z_{2} L_{2}$ for any $x$. Consequently, if $x^{k} \rightarrow 0$, then $m_{2}^{k} \rightarrow 0$ and so $N_{2}^{k} \rightarrow \infty$ and $n_{2}^{k} \rightarrow 1$. Also, if $n_{2}^{k} \rightarrow 1$, then $c_{2}^{k} \rightarrow 0$.

We argue that $p_{n n} \geq 1-\frac{\theta_{1}}{1-x}$. In the bargaining stage game player 1 does not opt-out in the first period. This is because if player 1 was opting-out in the first period, then the bargaining relationship is less valuable than being unmatched in the economy. This implies that $v_{1} \leq \delta_{1} v_{1}$, which is not possible. Player 2 can guarantee $1-\theta_{1}$ by immediately offering $\theta_{1}$ to player 1 . The best that player 2 can hope for is to receive 1 if there is no break-up and to receive $x$ if there is a break-up. Consequently,

$$
1-\theta_{1} \leq \operatorname{Pr}\{o p\} x+1-\operatorname{Pr}\{o p\}
$$

where $\operatorname{Pr}\{o p\}$ is the total probability of an opt-out. Hence, the total probability of an opt-out is at most $\frac{\theta_{1}}{1-x}$. So, $p_{n n}>1-\frac{\theta_{1}}{1-x}$.

Notice that implies that $N_{1} \leq \frac{\left(1-z_{1}\right) L_{1}}{p_{n n} n_{2}}$. However, because $n_{2}$ is close to 1 and $1-\frac{\theta_{1}}{1-x}>0$ for $x$ sufficiently small, $\bar{x}$ can be chosen such that for all $x<\bar{x}, n_{2} p_{n n}>$ $\xi>0$. So,

$$
c_{1} \geq \frac{z_{1} L_{1}}{z_{1} L_{1}+N_{1}} \geq \frac{\xi z_{1}}{\xi z_{1}+1-z_{1}}=\epsilon
$$

Step 4. If $x^{k}<\bar{x}$, then $\delta_{1} v_{1}\left(\sigma^{k}\right)>1-\theta_{2}$ for any equilibrium $\sigma^{k}$.

If $U_{1}(\sigma)>\theta_{1}-\kappa\left(1-e^{-r \Delta}\right)$, then for $x$ sufficiently small $v_{1}(\sigma) \geq n_{2} U_{1}(\sigma) \geq$ $\theta_{1}-\kappa\left(1-e^{-r \Delta}\right)$ since $n_{2}$ can be made arbitrarily close to 1 . In Appendix D we show $U_{1}(\sigma)>\theta_{1}-\kappa\left(1-e^{-r \Delta}\right)$ for all $x<\bar{x}$ and $\Delta<\bar{\Delta}$. For some intuition suppose that $\delta_{1} v_{1}(\sigma)<1-\theta_{2}$. If $\delta_{1} v_{1}(\sigma)<1-\theta_{2}$, then both agent's outside option is worse than yielding to their opponent so the bargaining stage-game satisfies the conditions of Lemma 2. If $c_{2}$ is arbitrarily close to zero, and $c_{1}$ is greater than $\epsilon$, then Lemma 2 implies that player 1's equilibrium bargaining game payoff $U_{1}(\sigma)$ is arbitrarily close to $\theta_{1}$. However, if $U_{1}(\sigma)$ is close to $\theta_{1}$, then so is $v_{1}(\sigma)$ contradicting that $\delta_{1} v_{1}(\sigma)<1-\theta_{2}$. 
Step 5. If $\delta_{1} v_{1}(\sigma)>1-\theta_{2}, \delta_{2} v_{2}(\sigma)=\delta_{2} x<1-\theta_{1}$, then the conditions of Lemma 1 are satisfied and the Lemma implies items ( $i)$ through (iii).

Theorem 1 considers a market with only one commitment type on each side. Suppose instead an agent of class $i$ is one of finitely many commitment types in set $T_{i}$. Let $\theta_{i}^{n}$ denote the inflexible demand of type $n$ of class $i$; let $z_{i}^{n}$ denote the fraction of class $i$ agents entering the market in each period who are of type $n$; and redefine $z_{i}=\sum_{n} z_{i}^{n}$. So, as before, $L_{i}\left(1-z_{i}\right)$ is the measure of rational agents of class $i$ entering the market in each period. Suppose that $\theta_{1}^{k}+\theta_{1}^{n}>2$ for any two commitment types $k$ and $n$. The following corollary shows that if the exogenous outside option $x$ is sufficiently small, then player 1 will mimic his most greedy commitment type and will receive a payoff arbitrarily close to the inflexible demand of his most greedy commitment type.

Corollary 1. Let $\bar{\theta}_{1}=\max _{\left\{n \in T_{1}\right\}}\left\{\theta_{1}^{n}\right\}$ and $\underline{\theta}_{2}=\min _{\left\{n \in T_{2}\right\}}\left\{\theta_{2}^{n}\right\}$. Suppose that $L_{2}(1-$ $\left.z_{2}\right)>L_{1}$ and $\delta_{1} \bar{\theta}_{1}>1-\underline{\theta}_{2}$. If $0 \leq \Delta<\bar{\Delta}$ and $0<x<\bar{x}$, then $v_{2}(\sigma)=x$ and $\delta_{1} v_{1}(\sigma)>1-\underline{\theta}_{2} ;$ and

(i) In the bargaining stage-game player 1 always proposes $\bar{\theta}_{1}$,

(ii) Player 2 reveals rationality in period 1 or period 2 ,

(iii) There exists a constant $\kappa>0$, that is independent of $\Delta$ such that,

$$
\begin{aligned}
\bar{\theta}_{1}-\kappa\left(1-e^{-r \Delta}\right) & \leq U_{1}(\sigma) \leq \bar{\theta}_{1} \\
1-\bar{\theta}_{1} & \leq U_{2}(\sigma) \leq 1-\bar{\theta}_{1}+\kappa\left(1-e^{-r \Delta}\right) \\
\bar{\theta}_{1}-\kappa\left(1-e^{-r \Delta}\right) & \leq v_{1}(\sigma)
\end{aligned}
$$

where $r=\max \left\{r_{1}, r_{2}\right\}$,

in any search equilibrium $\sigma$.

Proof. Let $C_{i}=\sum_{n \in T_{i}} C_{i}^{n}$ likewise $c_{i}=\sum_{n \in T_{i}} c_{i}^{n}$. The following are immediate consequences of Theorem [1] In any equilibrium $v_{2}(\sigma)=x$ and consequently $m_{2}<1$ and $m_{1}=1$. In any equilibrium $C_{1}^{n} \geq L_{1} z_{1}$ for any $n \in T_{1}$ and $C_{2}=z_{2} L_{2}$. Take a sequence of $x^{k} \rightarrow 0$ and let $\sigma^{k}$ denote a search equilibrium when the exogenous outside option is equal to $x^{k}$. For any sequence of search equilibria $\sigma^{k}, N_{2}^{k} \rightarrow \infty$, $n_{2}^{k} \rightarrow 1$ and $c_{2}^{k} \rightarrow 0$. Also, there exists $\epsilon>0$ such that, for all $x^{k}<\bar{x},\left(c_{1}^{n}\right)^{k} \geq \epsilon$ for any $n \in T_{1}$. 
We argue that if $x^{k}<\bar{x}$, then $\delta_{1} v_{1}\left(\sigma^{k}\right)>1-\underline{\theta}_{2}$ for any equilibrium $\sigma^{k}$.

If $U_{1}(\sigma)>\left(1-\xi\left|T_{2}\right|\right)\left(\bar{\theta}_{1}-\kappa\left(1-e^{-r \Delta}\right)-\xi\right)$, then for $x$ sufficiently small $v_{1}(\sigma) \geq$ $n_{2} U_{1}(\sigma) \geq\left(1-\xi\left|T_{2}\right|\right)\left(\bar{\theta}_{1}-\kappa\left(1-e^{-r \Delta}\right)-\xi\right)$ since $n_{2}$ can be made arbitrarily close to 1. Pick $\xi$ such that $\left(1-\xi\left|T_{2}\right|\right)\left(\bar{\theta}_{1}-\kappa\left(1-e^{-r \Delta}\right)-\xi\right)>1-\underline{\theta}_{2}$. Let $B \subset T_{2}$ denote the set of types for player 2 such that for any $n \in B$ the probability that player 2 mimics type $n$ is larger than $\xi$, conditional on player 1 mimicking $\bar{\theta}_{1}$ in period 1 , in equilibrium $\sigma$. Suppose that the set $B$ is non-empty. In any subgame where player 1 chooses to mimic $\bar{\theta}_{1}$ in period 1 and player 2 chooses to mimic $n \in B$ the argument for Theorem 1 Step 4, provided In Appendix D, implies that $U_{1}(\sigma)>\theta_{1}-\kappa\left(1-e^{-r \Delta}\right)-\xi$ for all $x<\bar{x}$ and $\Delta<\bar{\Delta}$.

Conditional on player 1 mimicking type $\bar{\theta}_{1}$ the probability that player 2 either mimics a type in $B$ or reveals rationality in period 1 or period 2 is at least $\left(1-\xi\left|T_{2}\right|\right)$ by the definition of the set $B$. If player 1 chooses $\bar{\theta}_{1}$ and player 2 reveals rationality then player 1 's payoff is at least $\bar{\theta}_{1}-\kappa\left(1-e^{-r \Delta}\right)$. Consequently, player 1 can secure payoff of at least $\left(1-\xi\left|T_{2}\right|\right)\left(\bar{\theta}_{1}-\kappa\left(1-e^{-r \Delta}\right)-\xi\right)$ by mimicking $\bar{\theta}_{1}$.

If $\delta_{1} v_{1}(\sigma)>1-\underline{\theta}_{2}, \delta_{2} v_{2}(\sigma)=\delta_{2} x<1-\bar{\theta}_{1}$, then player 1 can always choose to mimic type $\bar{\theta}_{1}$ by proposing $\bar{\theta}_{1}$ in period 1 . In the continuation game all the conditions of Lemma 1 are satisfied and the Lemma implies items $(i)$ through (iii).

5.2. Balanced Markets and Two-sided Reputation. In this subsection we focus on balanced markets, i.e., $L_{1}=L_{2}$. Recall that in a unbalanced market the equilibrium values for the long-side of the market are determined by market forces. More precisely, for a steady state to exist a portion of the long-side must voluntarily leave the market and so must receive value no more than $x$. In a balance market, one the other hand, flow demand and supply are equal and place no restrictions on the equilibrium values of agents. Consequently, a balance market leaves room for a richer set of outcomes in the bargaining stage-game.

The main result in this subsection, Theorem 2, shows that, in a balanced market, if the entering fraction of commitment types is unequal for the two sides $\left(z_{1} \neq z_{2}\right)$, then the endogenous outside option of the normal types must be compatible with the demands of the commitment types. Consequently, inefficiency in the market is substantial. 
Theorem 2. Suppose that $L_{2}=L_{1}=L$ and $z_{1}>z_{2}$. If $0<x<\bar{x}$, then for any equilibrium $\delta_{i} v_{i}(\sigma) \leq 1-\theta_{j}$ for $i \in\{1,2\}$.

The intuition for the result is as follows: Suppose that neither normal types trades with commitment types, which implies that $v_{i} \geq 1-\theta_{j}>x$ for $i \in\{1,2\}$, and so neither normal type leaves the market without trading. However, the assumption that normal types only trade with each other and $L\left(1-z_{1}\right) \neq L\left(1-z_{2}\right)$ makes a steady state impossible. Consequently, $v_{i} \leq 1-\theta_{j}$ for some $i \in\{1,2\}$. Suppose that player 1's value is strictly less than $1-\theta_{2}$; and player 2's value is strictly greater than $1-\theta_{1}$. This is exactly the situation covered by Lemma 1. So, in any equilibrium of the bargaining stage game player 1 trades with both the normal and the commitment type of player 2 with certainty. On the other hand player 2 only trades with the normal types of player 1 . This implies that for $x$ sufficiently small, the values of both the normal and commitment types of class 2 are good. Consequently, neither the normal nor the commitment types of class 1 will leave the market without trading. However, the commitment types of player 1 receive zero value in equilibrium and so leave the market voluntarily without trading. This implies that a flow $\left(1-z_{1}\right) L$ must accommodate the trades of flow $L$ which precludes a steady state.

Proof. Step 1. The normal types of class $i$ trade with the commitment types of class $j$, for some $i$, and consequently $\delta_{i} v_{i} \leq 1-\theta_{j}$

Suppose not, i.e., $p_{n c}=p_{c n}=0$. If $p_{n c}=p_{c n}=0$, then $\delta_{i} v_{i} \geq 1-\theta_{j}$ for all $i \in\{1,2\}$. Because $\delta_{i} v_{i} \geq 1-\theta_{j}>x$ the normal types will not leave the market voluntarily and all exit must occur through trade. The steady state equations imply:

$$
\begin{aligned}
& \left(1-z_{1}\right) L_{1}=N_{1} m_{1} n_{2} p_{n n} \\
& \left(1-z_{2}\right) L_{2}=N_{2} m_{2} n_{1} p_{n n}
\end{aligned}
$$

However, $N_{1} m_{1} n_{2} p_{n n}=N_{2} m_{2} n_{1} p_{n n}$ and $\left(1-z_{1}\right) L_{1} \neq\left(1-z_{2}\right) L_{2}$ leads to a contradiction.

Step 2. Step 1 implies that $\delta_{i} v_{i} \leq 1-\theta_{j}$ for some $i$. Suppose that $\delta_{i} v_{i}<1-\theta_{j}$ and $\delta_{j} v_{j}>1-\theta_{i}$. This configuration of outside options is covered by Lemma 1 which implies that both the normal type and the commitment type of player $j$ trade with certainty with the normal type of player $i$, i.e., $p_{c n}=p_{n n}=1$, and receive a payoff close the $\theta_{i}$ against the normal type of player $i$. However, this implies that the 
commitment types of player $j$ will only leave the market through trade with a normal player $i$ for sufficiently small $x$. The steady state equations for $j$ implies

(9+10)

$$
\begin{aligned}
\left(1-z_{j}\right) L & =m_{j} N_{j} p_{n n} n_{i} \\
z_{j} L & =m_{j} C_{j} p_{c n} n_{i} \\
L & =m_{j} n_{i}\left(N_{j} p_{n n}+C_{j} p_{c n}\right)
\end{aligned}
$$

The steady state equation for the normal type of class $i$ implies

$$
L\left(1-z_{i}\right)=m_{i} N_{i}\left(n_{j} p_{n n}+c_{j} p_{c n}\right)=m_{j} n_{i}\left(N_{j} p_{n n}+C_{j} p_{c n}\right)=L .
$$

However, $L \neq L z_{i}$, leading to a contradiction.

Step 3. Suppose that $\delta_{1} v_{1}=1-\theta_{2}$ and $\delta_{2} v_{2}>1-\theta_{1}$. This implies that the normal type of player 2 will never trade with the commitment type of player 1 . Also, the normal types of player 2 will only leave the market through trade since $\delta_{2} v_{2}>1-\theta_{1}>x$. So,

$$
\begin{aligned}
& \left(1-z_{2}\right) L=m_{2} N_{2} p_{n n} n_{1} \\
& \left(1-z_{1}\right) L \geq m_{1} N_{1} p_{n n} n_{2}=m_{2} N_{2} p_{n n} n_{1}
\end{aligned}
$$

However, this implies that $\left(1-z_{1}\right) L \geq\left(1-z_{2}\right) L$ which contradicts that $z_{1}>z_{2}$.

Step 4. Suppose that $\delta_{1} v_{1}>1-\theta_{2}$ and $\delta_{2} v_{2}=1-\theta_{1}$. The complete proof of this case is involved and so is given in Appendix E. A sketch is provided here. For a steady state to exists a portion of the commitment types of class 1 must leave the market without trading, i,e, their value from remaining in the market must be equal to $x$. To provide incentives for this $p_{c n}$ needs to be sufficiently small. However, if $p_{c n}$ is sufficiently small compared to $p_{n n}$, then the market is populated in large part by commitment types. This, however, would imply that player 2's payoff is also small and close to $x$, contradicting $\delta_{2} v_{2}=1-\theta_{1}$.

Equilibria in balanced markets contrast with both the equilibria in a market with complete information and equilibria in unbalanced markets (Theorem 1). In particular,

(i) The inflexible demands of the commitment types determine upper bounds on equilibrium values, i.e., $\delta_{1} v_{1} \leq 1-\theta_{2}$ and $\delta_{2} v_{2} \leq 1-\theta_{1}$. This implies that $v_{1}+v_{2}<1$ for $\delta_{i}$ close to one. Consequently, in a balanced market all 
equilibria entail significant inefficiency. In contrast in a market with complete information there is an efficient equilibrium where both player receiving their Rubinstein payoffs.

(ii) The inflexible demands of the commitment types $\left(\theta_{1}\right.$ and $\left.\theta_{2}\right)$ determine a lower bound on the magnitude of inefficiency in the market. This lower bound is independent of the entering proportion of commitment types. Hence inefficiency remains substantial even in the limiting case of complete rationality (i.e., for any small $z_{1}$ and $z_{2}$ ). This contrasts with models of two-sided incomplete information, such as Abreu and Gul (2000) or Compte and Jehiel (2002), where efficiency is restored in the limiting case of complete rationality.

(iii) There are a multitude of equilibria that entail two-sided reputation building. One such equilibria is constructed in Theorem 3. These equilibria all entail substantial delay, break-ups on the equilibrium path and inefficiency in the bargaining stage-game. This contrasts with both the market with complete information where bargaining is efficient and also with the unbalanced market where bargaining is asymptotically (for $\Delta$ small) efficient.

Theorem 11 restricts attention to the generic case where $z_{1} \neq z_{2}$. If the market is balanced and $z_{1}=z_{2}$, then an efficient equilibrium exists. In this efficient equilibrium normal agents receive their Rubinstein payoffs and the commitment types are never traded. However, there are also a multitude of inefficient equilibria 6

Theorem 2 considered a market with only one commitment type on each side. As in the case of unbalanced markets, the theorem can be extended to markets with multiple commitment types on each side. Suppose if $n<k$, then $\theta_{i}^{n}<\theta_{i}^{k}$, i.e, each classes commitment types are order according to increasing greediness. Suppose that $z_{2}>z_{1}$ and let $\tau_{1}$ denote the smallest index such that $1-z_{1}+\sum_{n=0}^{\tau_{1}} z_{1}^{n}=1-z_{2}$, if such a type exists. Note that $\tau_{1}$ is the least greedy commitment type that equate flow entry into the market by each side.

In the first part of the following corollary, we assume that the entry flow by class 2 normal types $\left(1-z_{2}\right)$ exceeds the entry flow by class 1 normal types $\left(1-z_{1}\right)$. Further we assume that the type space is rich enough so that there exists a type $\tau_{2}$ such that the total entry flow of class 2 agents less greedy than $\tau_{1}$ plus the normal types of class

\footnotetext{
${ }^{6}$ In particular, the inefficient equilibrium constructed in Theorem 3 remains an equilibrium. In the equilibrium constructed in the next section, inefficiency is due to delays and break-ups in the bargaining stage, not long queue lengths.
} 
$1\left(1-z_{1}+\sum_{n=1}^{\tau_{1}} z_{1}^{n}\right)$ equals the entry flow of class 2 normal types $\left(1-z_{2}\right)$. Under this assumption we show that an equilibrium exists where behavior is governed by one-sided reputation building in the bargaining stage and hence efficiency is restored. This market exhibits dynamics similar to the unbalanced market as characterized by corollary 1. We refer to this as the case of a "fine type space" since had the type distributions over commitment types been atomless with support $\left[\underline{\theta}_{1}, \bar{\theta}_{1}\right]$, then this condition would be automatically satisfied.

The second part of the corollary deals with the case of a coarse type space, that is, at least one commitment type is required on each side to equate the flow entry of the two sides $\left(\tau_{2}>0\right)$. In this case, the corollary shows that the findings of Theorem 1 remain valid, and the normal types on both sides are compatible with the demands of the cut-off commitment types. Consequently, inefficiency in the market remains substantial.

Corollary 2. Suppose that $L_{2}=L_{1}=L$ and $z_{1}<z_{2}$. Let $\underline{\theta}_{i}=\min _{n} \theta_{i}^{n}$.

(1) Fine type space and one-sided reputation. Assume that $\tau_{2}=0$. If $0 \leq \Delta<\bar{\Delta}$, $t_{\text {search }}<t^{*}$ and $0<x<\bar{x}$, then there exists an equilibrium where $\delta_{2} v_{2}(\sigma) \leq$ $1-\theta_{1}^{\tau_{1}}$ and $\delta_{1} v_{1}(\sigma)>1-\underline{\theta}_{2} ;$ and,

(a) In the bargaining stage-game player 1 always proposes $\theta_{1}^{\tau_{1}}$,

(b) Player 2 reveals rationality in period 1 or period 2 ,

(c) There exists a constant $\kappa>0$, that is independent of $\Delta$ such that,

$$
\begin{aligned}
\theta_{1}^{\tau_{1}}-\kappa\left(1-e^{-r \Delta}\right) & \leq U_{1}(\sigma) \leq \theta_{1}^{\tau_{1}} \\
1-\theta_{1}^{\tau_{1}} & \leq U_{2}(\sigma) \leq 1-\theta_{1}^{\tau_{1}}+\kappa\left(1-e^{-r \Delta}\right) \\
\theta_{1}^{\tau_{1}}-\kappa\left(1-e^{-r \Delta}\right) & \leq v_{1}(\sigma)
\end{aligned}
$$

(2) Coarse type space and two-sided reputation. Assume that $\tau_{2}>0$. If $0<x<\bar{x}$, then for any equilibrium $\delta_{i} v_{i}(\sigma) \leq 1-\underline{\theta}_{j}$ for all $i \in\{1,2\}$

Proof. The proof of part 1 is in the appendix, part 2 is an immediate consequence of Theorem 2 ,

5.3. An Inefficient Equilibrium with Selective Break-ups. As demonstrated in Theorem 2 all equilibria involve substantial inefficiency $\left(\delta_{i} v_{i} \leq 1-\theta_{j}\right)$. This inefficiency can result from two main sources. It can stem from a large queue length (i.e., a small market tightness parameter) for the side of the market that is strong 
in the bargaining stage-game. Alternatively, the overall inefficiency may result from inefficiency in the bargaining stage-game. The main result in this section, Theorem 3 , constructs an equilibrium where the market tightness parameter is equal to one. So, inefficiency does not stem from long queues. In this equilibrium, both agents build reputation in the bargaining stage game; and there are delays, resulting from a war of attrition a-la Abreu and Gul (2000), on the equilibrium path. Also, the two normal agents always trade with each other, one of the normal agents always trades with the commitment type and the other normal type opts-out with positive probability against the commitment type.

In order for a simpler exposition, the development that follows deals with the limiting case where the time between offers $\Delta=0$. The bargaining stage game is analyzed via a continuous time war of attrition. Also, we assume that $t_{\text {search }}=0$ and so $\delta_{i}=1$. The assumption $\Delta=0$ is non-essential and is used solely to simplify exposition. In Appendix $\mathrm{F}$ we validate the our use of continuous time by establishing that there exists a sequence of equilibria (Theorem 4) for games where $\Delta=\Delta^{n}$ and also that these equilibria converge to the continuous time characterization we discuss here as $\Delta_{n} \rightarrow 0$ and $t_{\text {search }}^{n} \rightarrow 0$ (Theorem 5 ).

5.3.1. The Bargaining Stage-Game as A War of Attrition with Opt-out. Each player chooses a real time $t \in[0, \infty)$ and an action $a \in\{$ Yield, Opt - out $\}=\{y, o\}$ that corresponds to the action at time $t$ conditional on his opponent not yielding or optingout before time $t$. A strategy is a plan of when to yield and when to opt-out conditional on one's opponent not yielding or opting out until that time. If player $i$ is the first player to yield at time $t$, his payoff from yielding is $\left(1-\theta_{j}\right) e^{-r_{i} t}$ and if at time $t$ player $j$ yields his payoff is $\theta_{i} e^{-r_{i} t}$. If both players yield at the same time $t$ player $i$ gets $e^{-r_{i} t}\left(\frac{\theta_{i}+1-\theta_{j}}{2}\right)$. If at time $t$ player $i$ yields and player $j$ opts-out, then players trade where player $i$ 's payoff is $\left(1-\theta_{j}\right) e^{-r_{i} t}$. The commitment type's (inflexible) strategy is to never yield to an opponent. A strategy for player $i$ can be identified by two cumulative distribution functions $F_{i}$ and $\alpha_{i}$ such $F_{i}(\infty)+\alpha_{i}(\infty) \leq 1$ where $F_{i}(t)$ is the probability with which player $i$ yields at or before time $t$, and $\alpha_{i}(t)$ is the probability with which player $i$ opts-out at or before time $t$.

5.3.2. Equilibrium. We term a search equilibrium "an equilibrium with selective break ups" (SBU Equilibrium) if 
(i) Values $v_{i}=1-\theta_{j}$ for both players,

(ii) If $\alpha_{i}(t)>0$ for some $t \geq 0$, then $\alpha_{j}(t)=0$ for all $t$,

(iii) If $\alpha_{i}(t)>0$ for some $t \geq 0$, then $F_{i}(t)=F_{i}\left(t^{\prime}\right)$ for any $t^{\prime}>t$.

The first condition is a restriction on equilibrium values and requires that in the bargaining stage-game players are indifferent between yielding and opting-out. The second condition requires that only player $i$ opts-out, and the third condition requires that if player $i$ opts-out with positive probability at some time $t$, then he does not yield at any time after $t$, including $t$. The following theorem shows that a SBU equilibria exist.

Theorem 3. Suppose that $z_{i}<z^{*}$. There exist a unique SBU equilibrium.

Proof. Suppose that player 1 is the player for whom $\alpha_{1}(t)>0$. In any SBU equilibrium, there exists a common time $T<\infty$ where both agents complete conceding to each other. There is a time $T$ such that player 1 only opts-out after time $T$. Hence, player 2 must complete conceding by time $T$ also. Also, $\alpha(t)$ must be concentrated at time $T$, since player 1 has no incentive to delay opting-out, after time T. Proposition 1, in Abreu and Gul (2000), implies that both agents must concede at rate $\lambda_{1}$ and $\lambda_{2}$. Consequently, in a SBU equilibrium $F_{2}(t)=1-b_{2} e^{-\lambda_{2} t}$, $F_{1}(t)=1-b_{1} e^{-\lambda_{1} t},\left(1-b_{1}\right)\left(1-b_{2}\right)=0$, and $b_{i} \in[0,1]$. The term $F_{i}(0)=1-b_{i}$ is the initial probability yielding for player $i$. Player $i$ 's equilibrium bargaining game payoff is $\left(1-\theta_{j}\right) b_{j}+\left(1-b_{j}\right) \theta_{i}$. So, condition $(i)$ implies that $b_{1}=b_{2}=1$. The fact that the players finish yielding and opting-out at some common time $T$ implies the following equation.

$$
\begin{aligned}
1-e^{-\lambda_{1} T} & =p_{n c} n_{1} \\
1-b_{2} e^{-\lambda_{2} T} & =n_{2}
\end{aligned}
$$

Equation (11) requires the probability that player 1 yields by time $T$ equal the probability that player 1 is the normal type. Equation (12) requires the probability that player 2 yields by time $T$ equal the probability that player 2 yields to a commitment type. In order, to show that a SBU equilibrium exists, we need to show that we can find a $p_{n c}$ such that $b_{2}=1$ and the previous equations are satisfied.

Note that, in this equilibrium, normal types trade if they are matched (since player 1 opts-out only after player 2 finishes yielding). Therefore $p_{c n}=p_{n n}=1, p_{c c}=0$ and 
$p_{n c} \in[0,1]$. Given these match probabilities the steady state equations are as follows

$$
\begin{aligned}
c_{2} & =\frac{z_{2}}{z_{2}+\left(1-z_{1}-z_{2}\right) p_{c n}} \\
n_{2} & =\frac{\left(1-z_{1}-z_{2}\right) p_{c n}}{z_{2}+\left(1-z_{1}-z_{2}\right) p_{c n}} \\
c_{1} & =\frac{z_{1}}{1-z_{2}} \\
n_{1} & =\frac{1-z_{1}-z_{2}}{1-z_{2}}
\end{aligned}
$$

Solving Equation (11) and Equation (12) for $b_{2}$ and substituting in for $n_{1}$ and $n_{2}$ from Equation (14) and Equation (16) gives $b_{2}$ as a function of $p_{n c}$

$$
b_{2}\left(p_{n c}\right)=\frac{z_{2}}{z_{2}+p_{n c}\left(1-z_{1}-z_{2}\right)}\left(\frac{1-z_{2}}{\left(1-z_{2}\right)-p_{n c}\left(1-z_{1}-z_{2}\right)}\right)^{\frac{\lambda_{2}}{\lambda_{1}}} .
$$

Let $k\left(p_{n c}\right)=z_{2}+p_{n c}\left(1-z_{1}-z_{2}\right)$. Consequently,

$$
b_{2}^{\lambda_{1}}=z_{2}^{\lambda_{1}}\left(1-z_{2}\right)^{\lambda_{2}} k\left(p_{n c}\right)^{-\lambda_{1}}\left(1-k\left(p_{n c}\right)\right)^{-\lambda_{2}} .
$$

Suppose that $p_{n n}=p_{c n}=p_{n c}=1$. This implies that no player opts-out in the bargaining stage-game, $c_{1}=\frac{z_{1}}{1-z_{2}}$ and $c_{2}=\frac{z_{2}}{1-z_{1}}$. Also, if player's yield to each other at the constant hazard rates $\lambda_{i}$, as defined by Equation (5), then without loss of generality, suppose that player 2 is the stronger player. Player 1 concedes with positive probability at time zero $1-b_{1}>0$ and $b_{2}=1$ where $b_{i}$ is defined as in Equation (8) . Observe that if $p_{n c}=1$, then $k(1)=1-z_{1}$ and $b_{2}(1)>1$ (recall player 2 is chosen as the stronger player when $\left.p_{n c}=p_{c n}=p_{n n}=1\right)$. If $p=0$, then $k(0)=z_{2}$ and $b_{1}=1$. Let $f(p)=z_{2}^{\lambda_{1}}\left(1-z_{2}\right)^{\lambda_{2}} k(p)^{-\lambda_{1}}(1-k(p))^{-\lambda_{2}}$. The function $f(k)$ is strictly convex and minimized at $k=\frac{\lambda_{1}}{\lambda_{2}+\lambda_{1}} \in(0,1)$. Pick $z^{*}$ such that $z^{*}<\frac{\lambda_{1}}{\lambda_{2}+\lambda_{1}}<1-z^{*}$. Let $p^{*}=\frac{\frac{\lambda_{1}}{\lambda_{1}+\lambda_{2}}-z_{2}}{1-z_{1}-z_{2}}$ so that $k\left(p^{*}\right)=\frac{\lambda_{1}}{\lambda_{2}+\lambda_{1}}$. Notice $b_{2}\left(p^{*}\right)<b_{2}(0)=1<b_{2}(1)$. If $z_{i}<z^{*}$, then there exists $p_{n c} \in\left(p^{*}, 1\right)$, such that $b_{2}\left(p_{n c}\right)=1=b_{2}^{*}$. Note that if we had chosen player 2 (i.e., the stronger player when $p_{n n}=p_{c n}=p_{n c}=1$ ) it is not possible to find $p_{\text {cn }}$ such that $b_{1}=b_{2}=1$.

5.4. The Limiting Case of Complete Rationality. We now turn to limit results as the (ex ante) probability of irrationality of both players go to zero. Note that neither Theorem 3 nor Theorem 2 requires a large fraction of commitment types in 
the market. The theorems only require that there is a strictly positive measure of commitment types in the entering population. The following corollary shows that for any sequence of SBU equilibria, the opt-out probability converges to zero and the steady state frequency of commitment types also converges to zero, as the entering fraction of commitment types goes to zero. However, even though asymptotically the market is free of commitment types, inefficiency is still substantial $\left(v_{i}=1-\theta_{j}\right.$ for both players).

Corollary 3. Suppose that $\lim z_{1}^{n}=\lim z_{2}^{n}=0$. For any sequence of SBU equilibria $\sigma^{n}$ such that, $c_{i}^{n} \rightarrow 0, p_{c n}^{n} \rightarrow 1$ and $p_{n c}^{n} \rightarrow 1$.

Proof. Let $i_{n}=\arg \max _{i=1,2}\left(z_{i, n}\right)^{\lambda_{j}}\left(1-z_{i, n}\right)^{\lambda_{i}}$. Then there is a unique solution for $z_{i, n}^{\lambda_{j}}\left(1-z_{i, n}\right)^{\lambda_{i}}=k_{n}^{\lambda_{j}}\left(1-k_{n}\right)^{\lambda_{i}}$ that satisfies $k_{n}>\frac{\lambda_{j}}{\lambda_{j}+\lambda_{i}}$. Since $z_{i, n} \rightarrow 0$, it should be that either $k_{n} \rightarrow 0$ or $k_{n} \rightarrow 1$. since $k_{n}>\frac{\lambda_{j}}{\lambda_{j}+\lambda_{i}}>0, k_{n} \rightarrow 1$. By definition $k_{n}=z_{i, n}+\left(1-z_{i, n}-z_{j, n}\right) p_{c n, n}$, therefore $p_{c n, n} \rightarrow 1$. Since $c_{i, n}=\frac{z_{i, n}}{k_{n}}, c_{i, n} \rightarrow 0$, and $c_{j, n}=\frac{z_{j, n}}{1-z_{i, n}} \rightarrow 0$ proving the claim.

Corollary 3 stands in sharp contrast to the literature. Abreu and Gul (2000) show that if there is some asymmetry on the two sides of the market (in particular if $\lambda_{1} \neq \lambda_{2}$ ), then as the commitment type probabilities approach to zero at the same rate, then the equilibrium payoff of the stronger side ( $i$ is the stronger side if $\lambda_{i}>\lambda_{j}$ ) approaches $\theta_{i}$ and the equilibrium payoff of the weaker side approaches $1-\theta_{i}$. Hence inefficiency disappears in the limit, but incomplete information still has an impact on the division of the surplus.

The reason for why inefficiency remains in our model even when the commitment types almost disappear is as follows. In the model when the agents are not allowed to opt-out, the only time a normal type can "use up" his probability is by yielding at time 0 . When the probability of commitment types approach zero, one side becomes infinitely stronger than the other, therefore the weaker player concedes with a huge probability at time 0 in order for the normal types to finish yielding at some common time $T$. In an SBU equilibrium, a player can use up his probability at time $T$ by opting-out. The player who would be the weaker player in the Abreu and Gul (2000) model (and hence would use up almost all his probability at time 0 by yielding) now uses up some of his probability by opting-out at time $T$, the period at which his opponent finishes yielding. Since in equilibrium yielding is via constant hazard rate, 
using up a constant of probability at time zero reduces the time to finish yielding much less than using up the same constant of probability at the end of yielding. Therefore, the amount of opt-out required to sustain this equilibrium does not go to 1 (and indeed goes to 0 ).

\section{Discussion}

We assumed that the commitment types evaluate their life-time utility from remaining in the market using an expected utility calculation identical to normal types. This implies that commitment types understand that their payoff from remaining in the market is worse than the payoff for the normal types. This assumption limits the impact of the commitment types on equilibrium outcomes, since commitment types that are rarely traded voluntarily leave the market. If, for example, commitment types were more optimistic about their prospects, then their impact would be even more pronounced.

In the model presented, we assumed that all agents are infinitely lived. An alternative modeling approach would be to incorporate a death rate. If a death rate is assumed, then the equilibrium where there is one-sided reputation building, as well as, the inefficient SBU-equilibrium would remain as equilibria. Also, if the death rate is sufficiently small, then all results presented in the paper remain unaltered.

\section{Appendix A. Proof of the Complete Information Benchmark}

Proof. Let $\bar{u}_{1}$ denote the best payoff player 1 receives in the bargaining stage game in any equilibrium. Consequently, $v_{i}(\sigma) \leq \bar{u}_{1}$ in any equilibrium $\sigma$. So, $\underline{u}_{2} \geq 1-e^{-r_{1} \Delta} \bar{u}_{1}$. In any period where player 2 proposes she can guarantee $1-e^{-r_{1} \Delta} \bar{u}_{1}$. In other words, player 2's worst payoff in a period she proposes $\underline{u}_{2} \geq 1-e^{-r_{1} \Delta} \bar{u}_{1}$. This is because in any period where player 1 responds he can expect at $\operatorname{most} \max \left\{e^{-r_{1} \Delta} \bar{u}_{1}, \delta_{1} v_{1}(\sigma)\right\}$ by rejecting the offer or opting-out. However, $\max \left\{e^{-r_{1} \Delta} \bar{u}_{1}, \delta_{1} v_{1}(\sigma)\right\}=e^{-r_{1} \Delta} \bar{u}_{1}$, because, $v_{i}(\sigma) \leq \bar{u}_{1}$ and $e^{-r_{1} \Delta} \geq \delta_{1}=e^{-r_{1} t_{\text {search }}}$.

Observe that $\bar{u}_{1} \leq 1-e^{-r_{2} \Delta} \underline{u}_{2}$. This is because player 2 can always reject player 1 's offer and guarantee $\underline{u}_{2}$. Consequently, we have the following system of inequalities 


$$
\begin{aligned}
& \bar{u}_{1} \leq 1-e^{-r_{2} \Delta} \underline{u}_{2} \\
& \underline{u}_{2} \geq 1-e^{-r_{1} \Delta} \bar{u}_{1} \\
& \bar{u}_{2} \leq 1-e^{-r_{1} \Delta} \underline{u}_{1} \\
& \underline{u}_{1} \geq 1-e^{-r_{2} \Delta} \bar{u}_{2}
\end{aligned}
$$

Solving this system implies that $U_{1}(\sigma)=\underline{u}_{1}=\bar{u}_{1}=u_{1}^{*}>v_{1}(\sigma)$ and $U_{2}(\sigma)=u_{2}^{*}$, in any equilibrium $\sigma$.

For item $(i)$ observe that in order for a steady state, some of the class $i$ agents must leave the market voluntarily and so $v_{i}(\sigma)=x$. Also,

$$
v_{i}(\sigma)=m_{i} u_{i}^{*}+\left(1-m_{i}\right) \delta_{i} v_{i}(\delta) \geq m_{i} u_{i}^{*} .
$$

Consequently, $u_{i}^{*}>x$ implies that $m_{i}<1$ and $m_{j}=1$. So, $v_{j}(\sigma)=u_{j}^{*}$.

Item $(i i): m_{i}=1$ for at least one of the agents $i$. Consequently, $v_{i}=u_{i}^{*}$ for this $i$. If $m_{i}=1$, then $m_{j} \leq 1$. Consequently, $v_{j} \leq u_{j}^{*}$. Also, it is easily verified that $m_{1}=m_{2}=1$ and $v_{i}(\sigma)=u_{i}^{*}$ is an equilibrium.

\section{Appendix B. Proof of Lemma 1}

Proof. Step 1. There exists a period $T$ such that in period $T+1$ player 2 is known to be the commitment type with certainty and the normal type of player 1 opts-out with certainty by time $T+1$.

For player 1 to not opt-out or not reveal rationality for another $K / \Delta$ periods the probability that player 2 concedes (i.e., reveals rationality by either offering something other than $1-\theta_{2}$ to player 1 , or by accepting $\left.1-\theta_{1}\right)$ to player 1 's demands, denoted $p$, must satisfy

$$
1-\theta_{2} \leq p+(1-p) e^{-r_{1} K}
$$

Player 1 can guarantee at least $1-\theta_{2}<\delta_{1} v_{1}(\sigma)$ today by opting out or by revealing rationality. Also, player 1 can hope for at most 1 if player 2 concedes, and player 1 can at most hope for a continuation payoff of 1 after the $K / \Delta$ periods. Choose $K$ sufficiently large so that $1-\theta_{2}>e^{-r_{1} K}$. So,

$$
1>p>\frac{1-\theta_{2}-e^{-r_{1} K}}{1-e^{-r_{1} K}}>0
$$


Choose, $N$ such that $\frac{c_{2}}{(1-p)^{N}}>1$. By period $N K / \Delta$ a rational player 1 is sure that player 2 is the commitment type in any equilibrium. Consequently, if $T+1>N K / \Delta$, then player 1 will opt-out with probability 1.

Step 2. If in history $h_{t}$ player 2 has revealed rationality by proposing something different than $\theta_{2}$ and player 1 is the commitment type with probability $\mu_{2}\left(h_{t}\right)>c_{1}$, then there exists a constant $\kappa>0$ such that,

$$
\begin{array}{r}
\theta-\kappa\left(1-e^{-r \Delta}\right) \leq U_{1}(\sigma) \leq \theta_{1} \\
1-\theta_{1} \leq U_{2}(\sigma) \leq 1-\theta_{1}+\kappa\left(1-e^{-r \Delta}\right) .
\end{array}
$$

Also, in any period where player 2 proposes, player 2's payoff is unique as a function of player 1's reputation level.

See Appendix C.

The steps that follow show that player 2 will either accept $\theta_{1}$ in period one, or will reveal rationality by proposing something other than $1-\theta_{2}$ in period two.

Step 3. If in an even period player 1 is known to be rational, then player 2 offers player 1 the Rubinstein payoff $e^{-r_{1} \Delta} u_{1}^{*}$, and player 1 accepts.

If player 2 asks for something other than $\theta_{2}$ and reveals rationality, then player 1 can reject the offer and secure the Rubinstein payoff $u_{1}^{*}$ in the next period. Consequently, in the event that player 2 asks for something other than $\theta_{2}$, then she will receive $1-e^{-r_{1} \Delta} u_{1}^{*}$.

Let $\bar{u}$ denote the highest continuation payoff for player 2 at the start of any period where she proposes for any reputation level. If player 2 asks for $\theta_{2}$, then player 1 will reject, because his outside option is larger than $\theta_{2}$. Also, in the next period where player 1 proposes he will never offer anything above $e^{-r_{2} \Delta} \bar{u}$. This is because player 1 will always opt-out against the commitment type and player 2 will always accept $e^{-r_{2} \Delta} \bar{u}$. So, player 2's best payoff if she proposes $\theta_{2}$ is $e^{-r_{2} 2 \Delta} \bar{u}$

But this implies that $\bar{u} \leq \max \left\{e^{-r_{2} 2 \Delta} \bar{u},\left(1-e^{-r_{1} \Delta}\right) u_{1}^{*}\right\}$ and so $\bar{u} \leq\left(1-e^{-r_{1} \Delta}\right) u_{1}^{*}$. Thus player 2 should reveal rationality and offer player 1 the Rubinstein split.

Step 4. If player 1 offers something different than $\theta_{1}$, then player 1 offers $u_{2}^{*}$.

If player 1 reveals rationality, then there will be an agreement in the next period and player 2 will receive $1-e^{-r_{1} \Delta} u_{1}^{*}$. Consequently, player 2 will accept $e^{-r_{2} \Delta}\left(1-e^{r_{1} \Delta} u_{1}^{*}\right)=$ $u_{2}^{*}$ and no less than this. So player 1 will offer $u_{2}^{*}$ player 2 and receive $u_{1}^{*}>e^{-r_{1} \Delta} u_{1}^{*}$ himself if he is to reveal rationality by deviating from $\theta_{1}$. 
Step 5. Let $S=\sup \left\{s: \exists h_{s}\right.$ s.t. $z_{i}\left(h_{s}\right) \geq c_{i}$ and $\operatorname{Pr}\left\{\right.$ player 1 proposes $u_{2}^{*}$ in period $\left.s \mid h_{s}\right\}>$ $0\}$. By Step $1 S \leq T$. Also, in period $T$, player 1 will only offer $\theta_{1}$. This is because the normal type of player 2 will always accept $\theta_{1}$ in period $T$ because player 1 will opt-out with certainty after period $T$.

Step 6. In period $S$ if player 1 instead offers $\theta_{1}$, then player 2's payoff after this offer is uniquely determined and is at least $1-\theta_{1}$ and is at most $1-\theta_{1}+\kappa\left(1-e^{-r \Delta}\right)$.

Once Player 2 reveals rationality her payoff is uniquely determined as a function of Player 1's reputation level. See, Lemma 5, Appendix C.

In the continuation game, player 2 either accepts $\theta_{1}$ in period $S$ or reveals rationality in period $S+1$. This is because player 1 only offers $\theta_{1}$ and never accepts $\theta_{2}$. Consequently, any trade occurs through player 2 either accepting $\theta_{1}$ or revealing rationality. However, player 1's reputation never decreases in the continuation. Also, player 2's continuation payoff, following player 2's revelation of rationality, is uniquely determined and decreasing in player 1's reputation level. So, player 2 does not gain anything from delaying revealing rationality after period $S+1$. Once Player 2 offers anything other than $\theta_{2}$, she is revealed as rational in period $S+1$, and player 1 can guarantee a continuation payoff equal to $e^{-r_{1} \Delta}\left(\theta_{1}-\kappa\left(1-e^{-r \Delta}\right)\right)$ (see Lemma 4. Appendix C). Hence, Player 2's payoff is at most $\left.1-\theta_{1}+(\kappa+1)\left(1-e^{-r \Delta}\right)\right)$. Also, player 2 can guarantee $1-\theta_{1}$ by simply accepting the offer in $S$.

Step 7. Player 1 prefers to offer $\theta_{1}$ instead of $u_{2}^{*}$ in period $S$. Consequently, Player 1 never offers anything but $\theta_{1}$.

If player 1 offers $u_{2}^{*}$, then the normal type player 2 will accept and if there is a rejection player 1 will opt-out in period $S+1$. If player 1 offers $\theta_{1}$ in period $S$, then normal player 2 will either accept or reveal rationality in period $S+1$. If player 2 does not reveal rationality, then player 1 will opt-out in period $S+1$. Consequently, player 1's payoff against the commitment type is identical regardless of whether he offers $\theta_{1}$ or $u_{2}^{*}$. Player 1's payoff once player 2 has revealed rationality is $\theta_{1}-\kappa\left(1-e^{-r \Delta}\right)$ by Lemma 1. So, if player 1 offers $\theta_{1}$, then her payoff against player 2 is at least $e^{-r_{1} \Delta}\left(\theta_{1}-\kappa\left(1-e^{-r \Delta}\right)\right.$ which exceeds $u_{1}^{*}$.

Step 8. Player 2 reveals rationality in period 2 (i.e., the first period she proposes) or accepts $\theta_{1}$ in period 1 . 
This is because player 1 always offers $\theta_{1}$. Consequently, there is no incentive for player 2 the delay revealing rationality. Since player 2 reveals either in the first period or the second period, player 1's payoff is at least $e^{-r_{1} \Delta}\left(\theta_{1}-\kappa\left(1-e^{-r \Delta}\right)\right.$.

\section{Appendix C. Equilibrium and Payoff functions in the game With ONE-SIDED INCOMPLETE INFORMATION}

In this section we summarize various findings by Myerson (1991), Abreu and Gul (2000), and Compte and Jehiel (2002) for the bargaining stage-game $\Gamma(\Delta, c, v)$, where $c_{1}>0, c_{2}=0$ and $\delta_{i} v_{i}<1-\theta_{j}$. For the continuation of the section assume that $c_{1}>0, c_{2}=0$ and $\delta_{i} v_{i}<1-\theta_{j}$. In words, we assume that player 1 is potentially the commitment type, player 2 is known to be rational with probability one, and both players' outside option is worse than yielding to the commitment type. Our development very closely follows Compte and Jehiel (2002) Appendix A where all the stated results can be found.

Lemma 4. Suppose that $c_{1}>0, c_{2}=0, \delta_{1} v_{1}<1-\theta_{2}$ and $\delta_{2} v_{2}<1-\theta_{1}$. There exists a constant $\kappa>0$, which is a function of $c_{1}, \theta_{1}$ and $\theta_{2}$, but that is independent of $\Delta$ such that,

$$
\begin{array}{r}
\theta-\kappa\left(1-e^{-r \Delta}\right) \leq U_{1}(\sigma) \leq \theta_{1} \\
1-\theta_{1} \leq U_{2}(\sigma) \leq 1-\theta_{1}+\kappa\left(1-e^{-r \Delta}\right)
\end{array}
$$

where $r=\max \left\{r_{1}, r_{2}\right\}$, in any perfect Bayesian equilibrium $\sigma$ of $\Gamma(\Delta, c, v)$.

Proof. See the development in Myerson (1991), Chapter 8, Theorem 8.4; or Abreu and Gul (2000) Lemma 1; or Compte and Jehiel (2002), Proposition 2.

Let $\gamma_{i}=e^{-r_{i} \Delta}$. Compte and Jehiel (2002), Appendix A, define

$$
\begin{aligned}
v^{n} & =\left[\gamma_{1}\right]^{2 n+1} \theta_{1} \\
\rho & =\frac{1-\left[\gamma_{1}\right]^{2}}{1-\left[\gamma_{2}\right]^{2}}, \pi^{0}=1-\mu^{0} \text { and } \\
w^{0}(\mu) & =\max \left\{(1-\mu)\left(1-v^{0}\right)+\mu \gamma_{2}\left(1-\theta_{1}\right), 1-\theta_{1}\right\} \\
\mu^{0} & : \gamma_{2} w^{0}\left(\mu^{0}\right)=1-\theta_{1} \text { if } \gamma_{2} w^{0}(0) \geq 1-\theta_{1}, \text { and } \mu^{0}=0 \text { otherwise. }
\end{aligned}
$$

Let $N$ be the largest integer for which 


$$
\left[\gamma_{1}\right]^{2 N} \theta_{1}>u_{2}^{*}(\Delta)
$$

Consider the sequence $\left\{\pi^{n}, \mu^{n}, w^{n}, w^{n}(.)\right\}_{0 \leq n \leq N}$ defined recursively by

$$
\begin{aligned}
\pi^{n+1} & =\frac{w^{n}}{w^{n}+\rho v^{n}}, \\
\mu^{n+1} & =\Pi_{k \leq n+1}\left(1-\pi^{k}\right), \\
w^{n+1} & =\pi^{n+1}\left(1+(\rho-1) v^{n}\right), \\
w^{n+1}(\mu) & =\left(1-\mu / \mu^{n}\right)\left(1-v^{n+1}\right)+\left(\mu / \mu^{n}\right)\left[\gamma_{2}\right]^{2} w^{n} .
\end{aligned}
$$

In this sequence $w^{n}=w^{n}\left(\mu^{n}\right)=w^{n-1}\left(\mu^{n}\right)$. The following lemma shows that player 2's equilibrium payoff is a continuous and non decreasing function of player 1's reputation level $\mu$. The strength of this lemma is that it shows player 2's equilibrium payoff is independent of which equilibrium is played and which history has been reached in the game. It is completely determined by player 1's level of reputation and by whether player 2 is a proposer or a responder.

Lemma 5. Suppose that $\mu>\mu^{N}$. Let $u_{2}(\mu)$ be the function that coincides with $w^{n}(\mu)$ on each interval $\left(\mu^{n+1}, \mu^{n}\right], n \in\{0, . ., N-1\}$.

(i) Player 1 proposes $\theta_{1}$ in all odd periods,

(ii) In any even period, if player 1's reputation level $\mu \in\left(\mu_{n+1}, \mu_{n}\right)$, then player 2 proposes $v_{n}$,

(iii) In any even period if player 1 is the commitment type with probability $\mu$, then player 2's equilibrium payoff is equal to $u_{2}(\mu)$,

(iv) In any odd period where player 1 has proposed $\theta_{1}$, if player 1 is the commitment type with probability $\mu$, then player 2's equilibrium payoff is equal to $\max \{1$ $\left.\theta_{1}, e^{-r_{2} \Delta} u_{2}(\mu)\right\}$

in any equilibrium $\sigma$ of $\Gamma(\Delta, c, v)$.

Proof. See Compte and Jehiel (2002), Proposition 10.

Remark 1. Note that $\mu^{N} \rightarrow 0$ as $\Delta \rightarrow 0$.

Observe that the previous lemma also pins down player 1's payoff at all point in the game except the cut-off reputation level $\mu_{n}$. This is because player 2 always offers $v_{n}$ when $\mu \in\left(\mu_{n+1}, \mu_{n}\right)$ and player 1 always randomizes between accepting and rejecting. 
Consequently, in any even period where player 1's reputation is $\mu \in\left(\mu_{n+1}, \mu_{n}\right)$, player 1 's equilibrium payoff is $v_{n}$. When $\mu=\mu_{n}$, player 2 may offer $v_{n}$ or $v_{n-1}$. Compte and Jehiel (2002) construct perfect bayesian equilibria where player 2 randomizes between $v_{n}$ and $v_{n-1}$ if $\mu=\mu_{n}$. Notice since there is an equilibria where player 2 randomizes between $v_{n}$ and $v_{n-1}$ there are also equilibria where player 2 chooses $v_{n}$ with certainty; chooses $v_{n+1}$ with certainty; or uses any randomization between these two offers. Consequently, if $\mu=\mu_{n}$, then player 1's equilibrium payoff set is the convex and closed interval $\left[v_{n}, v_{n-1}\right]$.

Lemma 6. There exists a perfect Bayesian Nash equilibrium for the game $\Gamma(\Delta, c, v)$. The equilibrium payoff set for player 1, viewed as a possibly multi-valued function of $\mu$ is an upper-hemi-continuous compact and convex valued correspondence.

Proof. See the above discussion and Compte and Jehiel (2002), Proposition 11.

\section{Appendix D. Proof Theorem 1, Step 4}

Consider the bargaining stage-game $\Gamma(\Delta, c, v)$. Suppose that $\delta_{2} v_{2}<1-\theta_{1}, \Delta<\bar{\Delta}$ and $c_{1}>\epsilon$. We'll show that for every $\xi$ there exists a $\bar{c}$ such that If $c_{2}<\bar{c}$, then $U_{1}(\sigma) \geq \theta_{1}-\kappa(\epsilon)\left(1-e^{-r_{2} \Delta}\right)-\xi$. In what follows revealing rationality means player $i$ either accepts something less than $\theta_{i}$ or proposes something other than $\theta_{i}$.

Step 1. There exists a period $T$, independent of $c_{2}$, such that if player 1 has not revealed rationality by period $T$, then he is known to be the commitment type with probability 1.

Follows immediately from Appendix B, Step 1.

Step 2. Player 2 must reveal rationality by period $T+1$.

If player 1 has not revealed rationality by time $T$, then player 2 knows that player 1 is the commitment type with probability 1 . So, player 2 will reveal rationality by at most period $T+1$.

Step 3. Let $p_{t}$ denote the total probability that player 2 reveals rationality in period $t$ by either accepting or proposing something other than $\theta_{2}$, after any history where $\mu_{1}\left(h_{t}\right) \geq c_{1}$. By the previous step and Bayes' rule

$$
\frac{c_{2}}{\prod_{t=1}^{T+1}\left(1-p_{t}\right)}=1
$$

Step 4. For every $\xi$, there exists a $\bar{c}$ such that if $c_{2}<\bar{c}$, then $p_{1}+p_{2} \geq 1-\xi$. 
Suppose not. Then there exists a sequence $c_{2}^{k} \rightarrow 0$ and a $\xi>0$ such that $p_{1}+p_{2}<$ $1-\xi$. This implies, by the previous step, that there exists $2<m \leq T+1$ and a subsequence of equilibria $\sigma^{k}$ such that $p_{m}^{k} \rightarrow 1$. If player 2 reveals rationality, then player 1's payoff is at least $\theta_{1}-\kappa\left(1-e^{-r \Delta}\right)$, by Lemma 4. Appendix C. Consequently, there exists a $K$ such that for all $k>K, 1-\theta_{2}<e^{-r_{1} \Delta} p_{m}^{k}\left(\theta_{1}-\kappa\left(1-e^{-r \Delta}\right)\right)$. This implies that player 1 will not reveal rationality in period $m-1$ in any equilibrium $\sigma^{k}$ for all $k>K$. Player 2's payoff at any even period, where player 2 is known to be rational with certainty, is a non-increasing function of the reputation level $\mu_{1}$ of player 1 . This is also true for all odd periods. In equilibrium $\sigma^{k} \mu_{1}\left(h_{m-2}\right) \leq \mu_{1}\left(h_{m}\right)$. Consequently, player 2's payoff from revealing rationality in period $m-2$ is strictly greater than player 2's payoff from not revealing rationality and then revealing rationality with probability $p_{m}^{k}$ in period $m$. However, this is a contradiction.

\section{Appendix E. Proof of Step 4, Theorem 2 and Corollary 2}

Proof of Step 4. In this case, player 1 and player 2 only leave the market through trade. So, for a steady state to exist the commitment types of class 1 should be willing to voluntarily leave the market.

Observe that the equilibrium value for player 1

$$
\begin{aligned}
& v_{1} \leq m_{1} p_{n n}+\delta_{1}\left(1-m_{1} p_{n n}\right) v_{1} \\
& v_{1} \leq \frac{p_{n n} m_{1}}{1-\delta_{1}\left(1-p_{n n} m_{1}\right)} \leq \frac{p_{n n} m_{1}}{1-\delta_{1}}
\end{aligned}
$$

So, $\delta_{1} v_{1}>1-\theta_{2}$ implies that $m_{1} \geq\left(1-\theta_{2}\right)\left(1-\delta_{1}\right)$ and $p_{n n} \geq\left(1-\theta_{2}\right)\left(1-\delta_{1}\right)$.

The following are the steady state equations for class 1 agents

$$
\begin{aligned}
N_{1} m_{1} n_{2} p_{n n} & =\left(1-z_{1}\right) L \\
C_{1} m_{1} n_{2} p_{c n} & =\left(z_{1}-z_{2}\right) L
\end{aligned}
$$

Let $\alpha=\frac{\left(z_{1}-z_{2}\right)}{\left(1-z_{1}\right)}$. Dividing the first equation by the second equation and using $n_{1}+c_{1}=1$ gives

$$
\begin{aligned}
n_{1} & =\frac{p_{c n}}{\alpha p_{n n}+p_{c n}} \\
c_{1} & =\frac{\alpha p_{n n}}{\alpha p_{n n}+p_{c n}}
\end{aligned}
$$


Consequently,

$$
c_{1} \geq \frac{\alpha\left(1-\delta_{1}\right)\left(1-\theta_{2}\right)}{\alpha\left(1-\delta_{1}\right)\left(1-\theta_{2}\right)+1} \equiv \underline{c} .
$$

If $c_{1} \geq \underline{c}>0$, then there exists time $T$, which is independent of $x$, such that the normal types trade or opt-out with probability 1 by time $T$. This is proved in Appendix B as a part of the proof of Lemma 1 .

If the commitment type of class 1 trades with Player 2, then the expected payoff to the commitment type conditional on trading is at least $e^{-r_{1} T} \theta_{1}$. This is because after time $T$ player 2 knows with certainty that her opponent is the commitment type. At this point player 2 will either immediately opt-out or immediately trade with the class 1 commitment type. Also, in any trade the commitment type of class 1 receives $\theta_{1}$.

In order for the steady state equations to hold the class 1 commitment types need to be indifferent between voluntarily leaving and remaining in the market. The commitment type receives at most $\theta_{1}$ from player 2 so

$$
\begin{aligned}
x & \geq m_{1} p_{c n} e^{-r_{1} T} \theta_{1}+\left(1-m_{1} p_{c n}\right) \delta_{1} x, \text { which implies } \\
p_{c n} & \leq \frac{\left(1-\delta_{1}\right) x}{m_{1}\left(e^{-r_{1} T} \theta_{1}-x \delta_{1}\right)}
\end{aligned}
$$

Combining the upper bound for $p_{c n}$ given in the above equation with steady state Equation (30) for $n_{1}$ implies

$$
n_{1} \leq \frac{\left(1-\delta_{1}\right) x}{\alpha p_{n n} m_{1}\left(e^{-r_{1} T} \theta_{1}-x \delta_{1}\right)+\left(1-\delta_{1}\right) x} \leq \frac{\left(1-\delta_{1}\right) x}{\alpha p_{n n} m_{1}\left(e^{-r_{1} T} \theta_{1}-x \delta_{1}\right)}
$$

The following gives a bound for player 2's equilibrium payoff:

$$
v_{2} \leq n_{1} p_{n n}+c_{1} p_{c n}\left(1-\theta_{1}\right)+\left(1-\left(n_{1} p_{n n}+c_{1} p_{c n}\right)\right) \delta_{2} v_{2}
$$

Equations (30) and (31) implies that $c_{1} p_{c n}=\alpha n_{1} p_{n n}$ substituting gives

$$
\begin{aligned}
& v_{2} \leq n_{1} p_{n n}\left(1+\alpha\left(1-\theta_{1}\right)\right)+\left(1-n_{1} p_{n n}(1+\alpha)\right) \delta_{2} v_{2} \\
& v_{2} \leq \frac{n_{1} p_{n n}\left(1+\alpha\left(1-\theta_{1}\right)\right)}{1-\delta_{2}+\delta n_{1} p_{n n}(1+\alpha)} \leq \frac{1+\alpha}{1-\delta_{2}} n_{1} p_{n n}
\end{aligned}
$$

Using the bounds on $n_{1}$ and $m_{1}$ implies

$$
v_{2} \leq \frac{1+\alpha}{1-\delta_{2}} \frac{x}{\alpha\left(1-\theta_{2}\right)\left(e^{-r_{1} T} \theta_{1}-x \delta_{1}\right)} \leq \frac{1+\alpha}{1-\delta_{2}} \frac{x}{\alpha\left(1-\theta_{2}\right)\left(e^{-r_{1} T} \theta_{1}-x \delta_{1}\right)}
$$


However if $x$ is sufficiently small, then the right hand is also small and $\delta_{2} v_{2}<1-\theta_{1}$ leading to a contradiction.

Proof of Corollary 2. Part 1. In this equilibrium player 2 never opts out against commitment types of player 1 that are less greedy than $\tau_{1}$, including $\tau_{1}$. Player 2 never trades with the commitment types greedier than $\tau_{1}$. Player 1's outside option $\delta_{1} v_{1}$ is strictly larger than yielding to any of player 2's commitment types. All commitment types of side 2 and all commitment types greedier that $\tau_{1}$ voluntarily leave the market since they are not traded.

In this case, the bargaining game is governed by Lemma 1. Consequently, player 1's payoff is at least $\underline{\theta}_{1}-\kappa(\Delta)$ and player 2's equilibrium value is at most $1-\underline{\theta}_{1}+\kappa(\Delta)$. Consequently, we can ensure that $\delta_{1} v_{1}>1-\min _{n} \theta_{2}^{n}$, by choosing $t_{\text {search }}$ and $\Delta$ sufficiently small. Also, pick the tightness parameter for side $1, m_{1}=1$. Again, it is straight forward to choose $t_{\text {search }}$ sufficiently small so that $\delta_{2} v_{2}$ is strictly greater than $\theta_{1}^{\tau_{1}+1}$. Also, pick $m_{2} \leq 1$ to ensure that $\delta_{2} v_{2} \leq \underline{\theta}_{1}$.

Part 2. Theorem 2 implies that $\delta_{i} v_{i} \leq 1-\min \theta_{j}$ for all $i$ and $j$.

\section{Appendix F. Existence And Convergence}

Let $f_{1}(t)$ denote the probability that player 1 reveals rationality in period $t$. A $\varsigma$-SBU equilibrium is a search equilibrium $\sigma$ with the following properties

(i) Player 2 trades with the commitment type with probability 1 ,

(ii) Player 1 opts-out with positive probability in a period $t$ only if $\sum_{s>t} f_{1}(s)<\varsigma$

(iii) $\delta_{1} v_{1}=1-\theta_{2}$ and $\delta_{2} v_{2}<1-\theta_{1}$.

\section{F.1. Existence.}

Theorem 4. Let $t_{\text {search }}=K \sqrt{\Delta}$ for some $K>\kappa$ where the constant $\kappa$ is defined as in Lemma 2, and suppose that $z^{*}$ is defined as in Theorem 3 . There exists $\Delta^{*}$ such that if, $\Delta<\Delta^{*}, z_{1}<z^{*}$ and $z_{2}<z^{*}$, then there exists $\varsigma(\Delta)$ such for all $\varsigma<\varsigma(\Delta)$ a $\varsigma-S B U$ equilibrium exists.

Proof. The proof defines an "alternative" bargaining game, proves that a search equilibrium exists if the players play this alternative game in the bargaining stage, and shows that this equilibrium is also an equilibrium for the original search economy.

Step 1. The alternative game. 
Given exogenous payoff function $w: \mathbb{N} \rightarrow \mathbb{R}^{2}$, exogenous total probability of breakup probability $a$, an outside options $v$, and vector of commitment type probabilities $c$ we define the alternative game $\hat{\Gamma}\left(a, c, v_{1}, w\right)$. In the alternative game player 1 moves first in the odd periods and player 2 moves first in the even periods. The player that moves first has two actions available, $\{R$ (eveal), $I$ (nsist) $\}$. If the player that moves first chooses $R$, then the game ends and payoffs are realized. If the player that moves first chooses $I$, then the follower picks action from $\{R$ (eveal), $I$ (nsist) $\}$. If she choses $R$, then the game ends and otherwise the game progresses to the next period. Also, at any node in period $t$ where player 1 moves the game ends with probability $\alpha_{1}(t)$. The opt-out probability $\alpha_{1}(t)$ is a function of $a$ and strategies and is defined in Step 3. The function $w: \mathbb{N} \rightarrow \mathbb{R}^{2}$ determines payoffs (before discounting) to each player from revealing and being revealed, at any period $t$, after a play of $R$ by the player who speaks first. In a period where $j$ moves first a typical element $w(t)=\left(w_{i}^{j}, w_{j}^{j}\right)(t)$ and $w_{i}^{j}(t)$ is the payoff to $i$ from $j$ revealing in period $t$. If the player that speaks second, player $j$, reveals, then player $i$ receives payoff $\theta_{i}$ and player $j$ receives payoff $1-\theta_{i}$. If there is a break-up in a period, then the agents receive $\delta_{i} v_{i}$ as their payoff. In this game the commitment types never opt-out or play reveal and player $i$ is the commitment type with probability $c_{i}$.

The alternative game is interpreted as follows: the strategy insist corresponds to player $i$ asking for $\theta_{i}$ and rejecting an offer of $\theta_{j}$ by player $j$ in the original game. Reveal corresponds to player $i$ proposing something different than $\theta_{1}$ but on an equilibrium path for the game with one-sided incomplete information in the original games. The exogenous continuation payoffs $w$ are chosen from the set of equilibrium payoff vectors for the game with one-sided incomplete information. The exogenously given opt-out probability $a$ is incorporated into the game so that player 1 only opts-out against the commitment type.

Step 2. Strategies in the alternative game. Let $\digamma=\{F: \mathbb{N} \rightarrow[0,1], F$ non-decreasing $\}$, that is $\digamma$ is the set of all sub-probability distribution functions over the set of natural numbers. Let $F(\infty)=\lim _{t \rightarrow \infty} F(t)$. Let $f$ denote the density of $F$, i.e., $f(t)=F(t)-F(t-1)$. A strategy for player 1 is a function $F_{1}$ such that $F_{1} \in \digamma$, and $\sum_{t} f_{1} \leq\left(1-c_{1}\right)(1-a)$. A strategy for player 2 is a function $F_{2} \in \digamma$ such that $\sum_{t} f_{2} \leq\left(1-c_{2}\right)$. 
Step 3. For any $F \in \digamma$ for player 1 let $t_{\varsigma}$ denote the first period such that $F(t) \geq F(\infty)-\varsigma$. For any exogenously given total opt-out probability a $\in[0,1]$ let

$$
\alpha_{1}(t, F, a)= \begin{cases}a \frac{F\left(t_{\varsigma}\right)-(F(\infty)-\varsigma)}{\varsigma} & \text { for } t=t_{\varsigma}, \\ a \frac{f(t)}{\varsigma} & \text { for } t>t_{\varsigma}, \\ 0 & \text { for } t<t_{\varsigma} .\end{cases}
$$

Step 4. Utilities in the alternative game

Suppose player $i$ uses strategy $F_{i}$. Define $\alpha_{2}()=$.0 . In the following we drop the dependence of $\alpha_{1}$ on $F_{1}$ and $a$ when this does not cause any ambiguity. In this game the payoff to player $i$ from revealing at time $t$ where player $i$ is the player to propose $U_{i}(F, a, c, v, w, t)=\sum_{s<t} \gamma_{i}^{s}\left(f_{j}(s) w_{i}^{j}(s)+\alpha_{j}(s) \delta_{i} v_{i}\right)+\left(1-F_{j}(t-1)-\sum_{s<t} \alpha_{j}(s)\right) \gamma_{i}^{t} w_{i}^{i}(t)$. The payoff to player $i$ from revealing at time $t$ where player $i$ is the player to respond $U_{i}(F, a, c, v, w, t)=\sum_{s \leq t} \gamma_{i}^{s}\left(f_{j}(s) w_{i}^{j}(s)+\alpha_{j}(s) \delta_{i} v_{i}\right)+\left(1-F_{j}(t)+\sum_{s \leq t} \alpha_{j}(s)\right) \gamma_{i}^{t}\left(1-\theta_{j}\right)$.

Step 5. The fixed point operator $\Phi$. 
Define correspondence $\Phi$ such that $\left(F^{\prime}, a^{\prime}, c^{\prime}, v^{\prime}, w^{\prime}\right) \in \Phi(F, a, c, v, w)$ if and only if

$$
\begin{aligned}
& a^{\prime}= \begin{cases}1-p^{*} & \text { if } \delta_{1} v_{1}<1-\theta_{2}, \\
0 & \text { if } \delta_{1} v_{1}>1-\theta_{2}, \\
{\left[0,1-p^{*}\right]} & \text { otherwise. }\end{cases} \\
& F_{1}^{\prime} \in \arg \max _{\left\{H_{1}: \sum_{t} h_{1}(t) \leq(1-a)\left(1-c_{1}\right)\right\}} \sum_{t \geq 0} \gamma_{1}^{t} h_{1}(t) U_{1}(F, a, c, v, w, t) \\
& F_{2}^{\prime} \in \arg \max _{\left\{H_{2}: \sum_{t} h_{2}(t) \leq 1-c_{2}\right\}} \sum_{t \geq 0} \gamma_{2}^{t} h_{2}(t) U_{2}(F, a, c, v, w, t) \\
& v_{1}^{\prime}=\sum_{t \geq 0} \gamma_{1}^{t}\left(\frac{f_{1}(t)}{1-c_{1}} U_{1}(F, a, c, v, w, t)+\frac{\alpha_{1}\left(t, F_{1}, a\right) \delta_{1} v_{1}}{1-c_{1}}\right) \\
& v_{2}^{\prime}=\min _{\left.\frac{1-\theta_{1}}{\delta_{2}}, \sum_{t \geq 0} \gamma_{2}^{t} \frac{f_{2}(t)}{1-c_{2}} U_{2}(F, a, c, v, w, t)\right\}}(F, a) z_{1} \\
& c_{1}^{\prime}=\frac{p(F, a) z_{2}}{p(F, a) z_{1}+1-\left(z_{1}+z_{2}\right)} \\
& c_{2}^{\prime}=\frac{\frac{p(F, a) z_{2}+\left(1-z_{1}-z_{2}\right)(1-a)}{p(F,}}{p}
\end{aligned}
$$

where $p^{*}=\frac{\frac{\lambda_{1}}{\lambda_{1}+\lambda_{2}}-z_{2}}{1-z_{1}-z_{2}}$ as defined in Theorem [3, $p(F, a)=\max \left\{p_{n n}(F, a), 1-\epsilon\right\}$ and $p_{n n}(F, a)$ denotes the probability that player 1 and player 2 trade, given revelation probabilities $F_{1}$ and $F_{2}$ and the opt-out probability $\alpha_{1}\left(F_{1}, a\right)$. The constant $\epsilon$ is chosen sufficiently small so that in the continuous time game considered in Theorem 3 , if $c_{1}$ and $c_{2}$ are calculated using $p_{n n}=1-\epsilon$ and $p_{n c}=p^{*}$, player 1 is the stronger player and $b_{2}\left(p^{*}, \epsilon\right)<1$.

Also, let $\mu_{i}(F, a, t)$ denote the probability that player $i$ is a commitment type given that player $i$ has not revealed rationality in history $h_{t}$. The posterior probability $\mu_{i}$ is obtained using Bayes' rule conditioning on strategies $\left(F_{1}, F_{2}, \alpha_{1}\left(F_{1}, a\right)\right)$. Notice $\mu_{i}(F, a, t)$ is a continuous function of $(F, a)$. Let

$$
\left(w_{i}^{j}, w_{j}^{j}\right)(t)^{\prime}=\left\{U_{i}\left(\mu_{i}(F, a, t)\right), U_{j}\left(\mu_{i}(F, a, t)\right)\right\},
$$

where $\left\{U_{i}\left(\mu_{i}(F, a, t)\right), U_{j}\left(\mu_{i}(F, a, t)\right)\right\}$ denotes the set of perfect equilibrium payoff vectors in the bargaining game with one-sided incomplete where player $i$ reputation level is $\mu_{i}(F, a, t)>0$. Recall that $\left(U_{1}\left(\mu_{1}\right), U_{2}\left(\mu_{1}\right)\right)$ is an upper-hemi continuous, 
convex and compact valued correspondence (as a function of $\mu_{1}$ ) by Lemma 6 in Appendix C.

The correspondence $\Phi$, as defined above is clearly upper hemi-continuous, compact and convex-valued (in the product topology). Consequently Glicksberg's fixed point theorem implies that a fixed point, $(F, a, c, v, w)$ exists.

Step 6. The remaining steps show that if $\varsigma$ and $\Delta$ are sufficiently small, then $\left(F, \alpha_{1}\left(F_{1}, a\right), c\right)$ is an equilibrium, and $v$ is the vector of values in this equilibrium, of the economy where the bargaining stage game is the original bargaining game and the continuation equilibrium once one player has revealed is chosen from the set of equilibria of the game with one-sided incomplete information such that payoffs are according to $w$.

Step 7. Player 1's value $\delta_{1} v_{1} \leq 1-\theta_{2}$. Also, $a>0$.

If $\delta_{1} v_{1}>1-\theta_{2}$, then $a=0$. If $a=0$, then this game is identical to the bargaining game without the options of opting-out. In this case, player 1 was chosen as the weaker player who concedes with probability $1-b_{1} \geq 0$. This implies that for $\Delta=0$ player 1's payoff in the bargaining stage is $1-\theta_{2}$. Consequently, by Lemma 2 , for $\Delta$ small $\delta_{1} v_{1}<1-\theta_{2}$. However, this implies that $a=1-p^{*}>0$, a contradiction.

Step 8. Player 2's equilibrium value $v_{2} \geq \frac{\left(1-\theta_{1}\right) c_{1}}{1-\left(1-c_{1}\right) \delta_{2}}$. This is because player 2 can always reveal immediately in period 1. This guarantees that player 2 will trade with the commitment type of class 1 in period 1 . So, $v_{2} \geq c_{1}\left(1-\theta_{1}\right)+\delta_{2} v_{2}\left(1-c_{1}\right)$. However, the formulation of the fixed point operator $\Phi$ implies that $c_{1} \geq \frac{z_{1} /(1-\epsilon)}{z_{1} /(1-\epsilon)+1-\left(z_{1}+z_{2}\right)}$. Consequently, $v_{2} \geq(1-\theta)-C\left(1-\delta_{2}\right)$ for some constant $C$ which is independent of $\Delta$.

Step 9. The probability that player 1 and player 2 trade, $p_{n n}(F, a, c) \geq 1-C \Delta$, where $C$ is a constant independent of $\Delta$. Consequently, commitment type probabilities $c_{1} \geq \underline{c}_{1}=\frac{z_{1} /(1-C \Delta)}{z_{1} /(1-C \Delta)+1-\left(z_{1}+z_{2}\right)}$ and $c_{2} \geq \underline{c}_{2}=\frac{z_{2} /(1-C \Delta)}{z_{2} /(1-C \Delta)+\left(1-z_{1}-z_{2}\right)}$.

Player 2 will complete her yielding by the latest in period $t_{\varsigma}$ for sufficiently small $\varsigma$. This is because the probability that player 1 yields by at most $\varsigma$ in any of these periods. Consequently, player 2 will do strictly better by completing yielding in period $t_{\varsigma}+1$ than in any period $t>t_{\varsigma}+1$.

Suppose that player 2 reveals rationality with probability $p>C \Delta$ in period $t_{\varsigma}$. Observe that player 1 reveals with positive probability in period $t_{\varsigma}$ by the definition of this period. 
Suppose that period $t_{\varsigma}$ is a period where player 1 is proposing. Instead of revealing rationality in $t_{\varsigma}$, player 1 can wait until $t_{\varsigma}+1$, and reveal rationality with certainty then, if player 2 has not revealed yet. This strategy can not do any better than revealing rationality in $t_{\varsigma}$. This implies that

$$
1-\theta_{2}+\Delta \kappa \geq \gamma_{1}(1-p)\left(1-\theta_{2}\right)+\theta_{1} p .
$$

This inequality cannot hold for $C$ sufficiently large.

Suppose that period $t_{\varsigma}$ is a period where player 1 is responding. However, since player 2 is revealing with probability $p$ in $t_{\varsigma}$ player 1 will not reveal in periods $t_{\varsigma}-1$ or $t_{\varsigma}-2$, if $C$ is sufficiently large. Since he does not reveal, his reputation level does not change in the two prior periods. This implies that player 2 is better of revealing in period $t_{\varsigma}-2$, if such a period exists. If such a period does not exists, this implies that player 1 is revealing with probability of at least $F_{1}(\infty)-\varsigma$ in period $t_{\varsigma}$. Notice for $\Delta$ small $\delta_{2}$ is close to 1 and so $\delta_{2} v_{2}$ is close $1-\theta_{2}$. This implies, however, that if player 1 is revealing with probability $F_{1}(\infty)-\varsigma$ in period $t_{\varsigma}$, then player 2 will do better by not revealing in period $t_{\varsigma}$.

Player 2 can not reveal with probability more than $C \Delta$ in period $t_{\varsigma}+1$ either. If player 2 was to reveal with probability greater than $C \Delta$, then player 1 will not reveal in period $t_{\varsigma}$. However, this contradicts the definition of period $t_{\varsigma}$ which requires that player 1 reveal with positive probability in this period. Consequently, the probability that player 2 reveals in period $t \geq t_{\varsigma}$ is at most $2 C \Delta$. Redefining $C$ implies that $p_{n n} \geq 1-C \Delta$.

Step 10. The bounds in the operator $\Phi$ are not binding, $a \in\left(0,1-p^{*}\right), \delta_{1} v_{1}=$ $1-\theta_{2}$, and $\delta_{2} v_{2}<1-\theta_{1}$. Consequently, $\left(F, \alpha_{1}\left(F_{1}, a\right), c\right)$ is an equilibrium, and $v$ is the vector of values in this equilibrium, of the economy where the bargaining stage game is the original bargaining game and the continuation equilibrium once one player has revealed is chosen from the set of equilibria of the game with one-sided incomplete information such that payoffs are according to $w$.

Suppose that $\delta_{1} v_{1}<1-\theta_{2}$, then $a=1-p^{*}$. Revelations need to occur at rate $\lambda_{1}$ and $\lambda_{2}$ by Lemma 2. However, $p^{*}$ is chosen such that if revelations occur at rate $\lambda_{1}$ and $\lambda_{2}$, then for $\Delta$ small player 2 is the player that reveals with a jump in the first two periods. Moreover, player 2's probability of revelation approaches $1-b_{2}>0$. This would imply that $\delta_{1} v_{1}>1-\theta_{2}$ for sufficiently small $\Delta$. Consequently, $\delta_{1} v_{1}=1-\theta_{2}$ 
and $a \in\left(0,1-p^{*}\right)$. Notice that since $t_{\text {search }}$ is larger than $\kappa \sqrt{\Delta}$, player 2 needs to be the player that reveals rationality with a jump in the first two periods, i.e., $b_{2}<1$ and $b_{1}=1$ as defined in Lemma 2. This however, implies that player 2's value $\delta_{2} v_{2}<1-\theta_{2}$.

\section{F.2. Convergence of SBU equilibrium in discrete time games to a corre- sponding SBU equilibrium of the war of attrition game. Let $z^{*}, \Delta^{*}$ denote} the constants specified in Theorem 4 . Suppose that $z_{1}, z_{2}<z^{*}$. Let $\left\{\sigma^{n}\right\}$ denote a sequence of $\varsigma$-SBU equilibrium for the economy where the period length is $\Delta^{*}>\Delta^{n}>0$. Suppose $\lim _{n \rightarrow \infty} \Delta_{n}=0$ and consequently $t_{\text {search }}^{n} \rightarrow 0$. Such a sequence of equilibria exists be Theorem 4 .

Remark 2. Let $\left(F_{1}^{n}, \alpha^{n}, F_{2}^{n}, v^{n}, c^{n}\right)$ denote a SBU equilibrium for $\Delta^{n}$. By construction $c_{i}^{n} \geq \underline{c}_{i}$. By Step 1, of Appendix $\square$ there exists a time $T$ such that $\frac{F(T)^{n}}{1-\underline{c}_{1}}+$

$\sum_{t \leq T} \alpha(t)^{n}=1$ and $\frac{F_{2}^{n}(T)}{1-\underline{c}_{2}}=1$, for all $n$. Hence the sub-probability distributions $\left(F_{1}^{n}, \alpha^{n}, F_{2}^{n}\right)$ have uniformly bounded support $[0, T]$. Consequently, by Helly's theorem, (Billingsley (1995), Theorem 25.9) implies that $\left(F_{1}^{n}, \alpha^{n}, F_{2}^{n}, v^{n}, c^{n}\right)$ has a convergent subsequence. Let $\left(F_{1}, \alpha, F_{2}, v, c\right)$ denote such a sub-sequential limit.

Theorem 5. $\left(F_{1}, \alpha, F_{2}\right)$ comprise a SBU equilibrium for the continuous time bargaining stage-game where the vector of commitment type probabilities is $c, v$ is the equilibrium values given that the $S B U$ equilibrium $\left(F_{1}, \alpha, F_{2}\right)$ is played in the bargaining stage-game, and the vector $c$ satisfies the steady state equations.

Proof. Step 1. The vector $c^{n}$ and $a^{n}$ satisfy the steady state equations for all $n$, consequently, $c$ and $a$ satisfies the steady state equations. The values $\delta_{1} v_{1}^{n}=1-\theta_{2}$ and $\delta_{2} v_{2}^{n}=1-\theta_{1}$ for all $n$, consequently $\delta_{1} v_{1}^{n}=1-\theta_{2}$ and $\delta_{2} v_{2}^{n} \leq 1-\theta_{1}$.

Step 2. $F_{1}$ and $F_{2}$ do not have common discontinuity points. Also, $G=F_{1}+\alpha$ and $F_{2}$ do not have common discontinuity points.

Step 3. Let $U_{1}^{n}=\iint U_{1}(t, k) d G^{n}(t) d F_{2}^{n}(k)$ and $U_{2}^{n}=\iint U_{2}(t, k) d F_{1}^{n}(t) d F_{2}^{n}(k)$ where

$$
U_{i}(t, k)= \begin{cases}\theta_{i} & \text { if } t>k, \\ 1-\theta_{j} & \text { if } t<k \\ 1 / 2 & \text { if } t=k .\end{cases}
$$


$F_{1}, F_{2}$ and $G$ do not have common discontinuity points consequently Billingsley (1995) Theorem 29.2 and Exercise 29.2 implies that $\lim U_{1}^{n}=\iint U_{1}(t, k) d G(t) d F_{2}(k)$ and $\lim U_{2}^{n}=\iint U_{2}(t, k) d F_{1}(t) d F_{2}(k)$. Also, $v_{1}=\frac{\lim U_{1}^{n}}{1-c_{1}}$ and $v_{2}=\frac{\lim U_{2}^{n}}{1-c_{2}}$.

Step 4. The functions $\left(F_{1}, \alpha, F_{2}\right)$ comprise a SBU equilibrium for the continuous time war of attrition with opt-outs.

$F_{1}$ does not jump at $T$ and $p_{n c}>p^{*}$ by construction.

In the continuous time war of attrition, if player 1 is behaving according to $F_{1}, \alpha$, then for each $\epsilon$, there is a $N$ such that for all $n>N, F_{2}^{n}$ is an $\epsilon$ best response to $F_{1}, \alpha$ and consequently, since $\epsilon$ is arbitrary $F_{2}$ is a best response to $F_{1}, \alpha$. Also, the symmetric argument is true for player 2 showing that $F_{1}, \alpha$ is a best response to $F_{2}$. Proving that $F_{1}, \alpha$ and $F_{2}$ form an equilibrium for the continuous time war of attrition. Since the war of attrition has a unique equilibrium with $p_{n c}>p^{*}, F_{1}, \alpha$ and $F_{2}$ coincide with this equilibrium. This argument is identical to Abreu and Gul (2000), proof of Proposition 4, on page 114 where a more detailed proof may be found.

\section{REFERENCES}

Abreu, D., And F. Gul (2000): "Bargaining and Reputation," Econometrica, 68(1), 85-117.

Abreu, D., AND D. PeArCE (2007): "Bargaining, reputation, and equilibrium selection in repeated games with contracts," Econometrica, 75(3), 653-710.

Billingsley, P. (1995): Probability and Measure. John Wiley and Sons, New York, NY.

Chatterjee, K., AND L. SAmuelson (1987): "Bargaining with two-sided incomplete information: An infinite horizon model with alternating offers," Review of Economic Studies, 54(2), 175-192.

Compte, O., AND P. Jehiel (2002): "On the Role of outside Options in Bargaining with Obstinate Parties," Econometrica, 70(4), 1477-1517.

FudenberG, D., AND D. Levine (1989): "Reputation and equilibrium selection in games with a patient player," Econometrica, 57, 759-778.

- (1992): "Maintaining a reputation when strategies are imperfectly observed," Review of Economic Studies, 59, 561-579.

Kambe, S. (1999): "Bargaining with Imperfect Commitment," Games and Economic Behavior, $28(2), 217-237$.

Kreps, D., AND R. Wilson (1982): "Reputation and Imperfect Information," Journal of Economic Theory, 27, 253-279.

Mailath, G., And L. Samuelson (2006): Repeated Games and Reputations: Long-run Relationships. Oxford University Press, Oxford, UK. 
Milgrom, P., And J. Roberts (1982): "Predation, Reputation, and Entry Deterrence," Journal of Economic Theory, 27, 280-312.

Myerson, R. (1991): Game Theory: Analysis of Conflict. Harvard University Press.

Osborne, M., And A. Rubinstein (1990): Bargaining and markets. Academic Press San Diego. Perry, M., AND P. Reny (1993): "A Non-cooperative Bargaining Model with Strategically Timed Offers," Journal of Economic Theory, 59, 50-50.

Rubinstein, A. (1982): "Perfect Equilibrium in a Bargaining Model," Econometrica, 50(1), 97-109. Rubinstein, A., And A. Wolinsky (1985): "Equilibrium in a Market with Sequential Bargaining," Econometrica, 53(5), 1133-1150.

— (1990): "Decentralized Trading, Strategic Behaviour and the Walrasian Outcome," The Review of Economic Studies, 57(1), 63-78.

Serrano, R., And O. Yosha (1993): "Information revelation in a market with pairwise meetings: the one sided information case," Economic Theory, 3(3), 481-499.

Shaked, A., AND J. Sutton (1984): "Involuntary Unemployment as a Perfect Equilibrium in a Bargaining Model," Econometrica, 52(6), 1351-1364.

Sutton, J. (1986): "Non-Cooperative Bargaining Theory: An Introduction," Review of Economic Studies, 53, 709-24.

Watson, J. (1998): "Alternating-Offer Bargaining with Two-Sided Incomplete Information," The Review of Economic Studies, 65(3), 573-594.

Meds, Kellogg School of Management, Northwestern University, 2001 Sheridan RoAd, Evanston, IL 60208

E-mail address: a-atakan@kellogg.northwestern.edu

E-mail address: m-ekmekci@kellogg.northwestern.edu 